\title{
IMM-H004 Protects against Cerebral Ischemia Injury and Cardiopulmonary Complications via CKLF1 Mediated Inflammation Pathway in Adult and Aged Rats
}

\author{
Qidi Ai ${ }^{1,2}$, Chen Chen ${ }^{2}$, Shifeng Chu ${ }^{2}$, Yun Luo ${ }^{3}$, Zhao Zhang ${ }^{2}$, Shuai Zhang ${ }^{2}$, Pengfei Yang ${ }^{2}$, \\ Yan Gao ${ }^{2}$, Xiaoling Zhang ${ }^{2}$ and Naihong Chen ${ }^{1,2, * \mathbb{D}}$ \\ 1 Hunan Engineering Technology Center of Standardization and Function of Chinese Herbal Decoction \\ Pieces \& College of Pharmacy, Hunan University of Chinese Medicine, Changsha 410208, China; \\ 13357329630@163.com \\ 2 State Key Laboratory of Bioactive Substances and Functions of Natural Medicines, Institute of Materia \\ Medica \& Neuroscience Center, Chinese Academy of Medical Sciences and Peking Union Medical College, \\ Beijing 100050, China; chenchen@imm.ac.cn (C.C.); chushifeng@imm.ac.cn (S.C.); \\ zhangzhao@imm.ac.cn (Z.Z.); zhangshuai2586@163.com (S.Z.); yangclatter@163.com (P.Y.); \\ gaoyantalentback@126.com (Y.G.); zxl123456mm@163.com (X.Z.) \\ 3 Institute of Medicinal Plant Development, Peking Union Medical College and Chinese Academy of \\ Medical Sciences, Beijing 100193, China; ly20040423@126.com \\ * Correspondence: chennh@imm.ac.cn; Tel.: +86-10-6316-5177
}

Received: 19 February 2019; Accepted: 2 April 2019; Published: 3 April 2019

\begin{abstract}
Background: Chemokine-like factor 1 (CKLF1) is a chemokine with potential to be a target for stroke therapy. Compound IMM-H004 is a novel coumarin derivative screened from a CKLF1/C-C chemokine receptor type 4 (CCR4) system and has been reported to improve cerebral ischemia/reperfusion injury. This study aims to investigate the protective effects of IMM-H004 on cerebral ischemia injury and its infectious cardiopulmonary complications in adult and aged rats from the CKLF1 perspective. (2) Methods: The effects of IMM-H004 on the protection was determined by 2,3,5-triphenyltetrazolium chloride (TTC) staining, behavior tests, magnetic resonance imaging (MRI) scans, enzyme-linked immunosorbent assay (ELISA), Nissl staining, histo-pathological examination, and cardiopulmonary function detection. Immunohistological staining, immunofluorescence staining, quantitative real-time PCR (qPCR), and western blotting were used to elucidate the underlying mechanisms. (3) Results: IMM-H004 protects against cerebral ischemia induced brain injury and its cardiopulmonary complications, inhibiting injury, and inflammation through CKLF1-dependent anti-inflammation pathway in adult and aged rats. IMM-H004 downregulates the amount of CKLF1, suppressing the followed inflammatory response, and further protects the damaged organs from ischemic injury. (4) Conclusions: The present study suggested that the protective mechanism of IMM-H004 is dependent on CKLF1, which will lead to excessive inflammatory response in cerebral ischemia. IMM-H004 could also be a therapeutic agent in therapy for ischemic stroke and cardiopulmonary complications in the aged population.
\end{abstract}

Keywords: IMM-H004; cerebral ischemia; complication; CKLF1; inflammation; aged rats

\section{Introduction}

Acute ischemic stroke is a devastating and debilitating disease, leading to high morbidity and mortality worldwide [1]. Aging is the biggest risk factor of stroke [2], with incidence doubled every decade after 55 years old [3]. The International Stroke Trial, including more than 17,000 stroke patients, 
revealed $84.1 \%$ of enrolled subjects were over 60 years old [4]. The average age of first stroke onset was at about 70 years old in very large reports, highlighting the large aged population of stroke patients [5,6].

Thrombolytic therapy is commonly used in patients with acute ischemic stroke, and recombinant tissue plasminogen activator (tPA) is the only Food and Drug Administration (FDA)-approved drug in clinical application. However, it is widely known that thrombolytic therapy only beneficial within $4.5 \mathrm{~h}$ after ischemic onset [7], but also with high incidences of side effects—-such as causing hemorrhagic transformation or augmenting the inflammatory response $[8,9]$. In present clinical practice, many patients cannot be administered tPA for exceeding the narrow time window, resulting in permanent cerebral ischemic injury. Therefore, it is necessary and urgent to develop safer and more effective therapeutic agents for ischemic stroke in the aged population.

Post-stroke complications are major contributors to the high mortality rate of ischemic stroke $[10,11]$. Long-term clinical observations have indicated that the complications are concentrating on heart and lung organ dysfunctions, including myocardial infarction (MI), pulmonary embolism $(\mathrm{PE})$, and pneumonia, which are also the most common causes for ischemic stroke death [10-15]. Cardiovascular disease is the main cause of death from the second to the fifth year after stroke [16,17]. The immune system is known to participate in ischemic brain injury, and the most common infection after stroke is pneumonia, which has a three-fold increase in the risk of death [18-23]. In addition, the aged population is more prone to post-stroke complications due to the weaker immune system compared with young people [1]. However, there are no effective drugs available for both cerebral injury and complications in ischemic stroke therapy to date. Research of ischemic stroke treatment mainly focuses on protection against brain injury but few on post-stroke complications, thus, developing novel drugs with multiple function for cerebral damage and cardiopulmonary complications is extremely important.

Chemokine-like factor 1 (CKLF1) is a chemokine with C-C chemokine receptor type 4 (CCR4) as a receptor. Current research mainly focuses on the role of CKLF1 in periphery infectious diseases [24-27]. Our previous studies have found a significant increase of CKLF1 expression in damaged brain as early as $12 \mathrm{~h}$ after middle cerebral artery occlusion (tMCAO), which peaks at day 2 after tMCAO in cerebral ischemic rats [28]. Intracerebroventricular-targeted injection of C19, an antagonist peptide of CKLF1, can improve cerebral ischemia injury in rats [29]. In addition, administration of anti-CKLF1 antibody also shows beneficial effects on smaller infarctions and better neurological behavior in rats [30].

Although the mechanism of acute ischemic stroke induced pneumonia and cardiovascular complications is still unclear, studies have shown that it may have a close association with inflammation [31,32]. As a chemokine, CKLF1 may play an important role in immune response after stroke. Thus, whether CKLF1 involves in the stroke induced cardiopulmonary complications is of considerable interest. IMM-H004 is a novel 3-piperazinylcoumarin small molecule compound screened from a CKLF1/CCR4 system by calcium transient technology and modified [27,33]. Our previous work has demonstrated protective effects of IMM-H004 on cerebral ischemia. It can protect against global cerebral ischemia by suppressing apoptosis and maintaining the integrity of synaptic structure in adult rats [34], improving BBB function via inhibiting the release of inflammatory factors in mice $[35,36]$. IMM-H004 can also significantly ameliorate cerebral ischemia/reperfusion-caused injury and subsequent inflammation in spontaneously hypertensive rats [37]. Additionally, IMM-H004 could reduce tPA's side effects by improving energy metabolism when combined with tPA [38]. However, it is undetermined whether IMM-H004 has curative effect on aged rats with permanent cerebral ischemia injury and especially the accompanied complications. There are also no studies investigating the change of CKLF1 when administered with IMM-H004. Therefore, our aim in the present study is to determine the therapeutic effects of IMM-H004 on permanent focal cerebral ischemia and cardiopulmonary complications in adult and aged rats, elucidating the underlying mechanisms from the CKLF1 pathway. 


\section{Results}

\subsection{Effects of IMM-H004 on Permanent Focal Cerebral Ischemia-Induced Brain Injury in Adult Rats}

The protective effect of IMM-H004 on permanent focal cerebral ischemia-induced brain injury is determined. Firstly, the therapeutic time window of IMM-H004 was investigated. The infarct size of a representative rat assessed by the TTC assay is shown in Figure $1 \mathrm{C}$. Compared with the pMCAO-operated group, rats administered with IMM-H004 (10 mg/kg) showed smaller brain infarct size and ameliorated neurological deficits pronouncedly at $3 \mathrm{~h}$ and $6 \mathrm{~h}$ after ischemia, but IMM-H004 administration showed no protective effect at $9 \mathrm{~h}$ and $12 \mathrm{~h}$ after ischemia. These results showed that IMM-H004 $(10 \mathrm{mg} / \mathrm{kg})$ treatment could decrease permanent focal cerebral ischemia-induced brain injury, and its therapeutic time window for ischemia is 0 to $6 \mathrm{~h}$ (Figure 1C,D). In order to exclude the impact of IMM-004 on the sham group, we set up the sham combined with IMM-H004 group to compare with the sham group. The results showed that there were no significant differences between the sham group and sham combined with IMM-H004 group in parameters we checked (infarction by TTC staining, neurobehavioral deficit tests, and inflammatory cytokines by ELISA analysis) (Figures S1 and S2).

A<smiles>C=C1Oc2cc(O)cc(OC)c2C(C)=C1N1CCN(C)CC1</smiles>

B

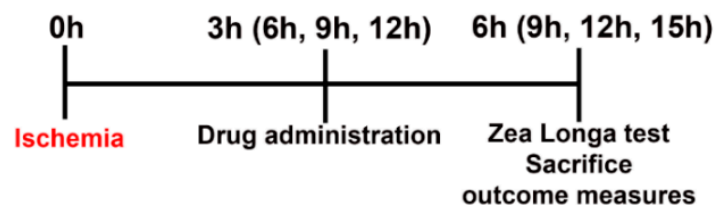

C

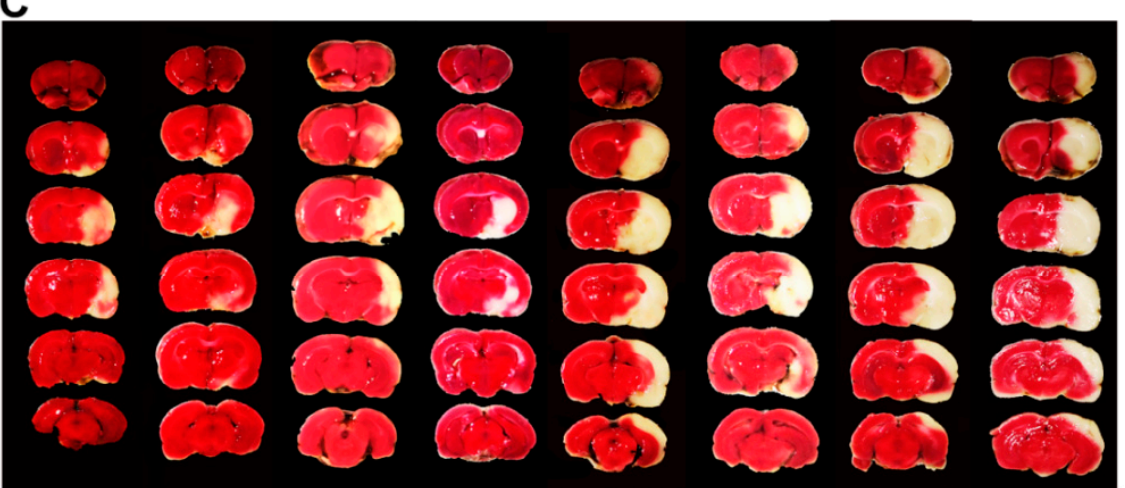

Model IMM-H004 Model IMM-H004 Model IMM-H004 Model IMM-H004

3 hours

6 hours

9 hours

12 hours

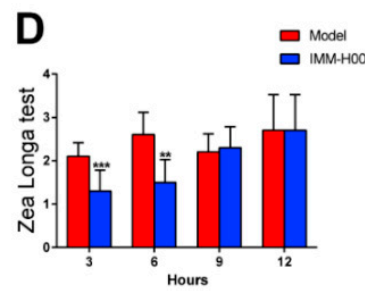

Figure 1. Therapeutic time window experiment of IMM-H004. (A) The chemical structure of IMM-H004. (B) The timeline diagram of theraputic time window experiment. (C) Representative images of TTC staining, and analysis of infarction area and edema ratio. (D) Statistical analysis of Zea Longa test scores. Data are shown as the mean $\pm \operatorname{SD}$ ( $n=10$ /group). ${ }^{* *} p<0.01$ vs. model; ${ }^{* * *} p<0.001$ vs. model.

Secondly, we determined the therapeutic dosage window of IMM-H004. Representative TTC staining for brains of rats administrated with different dosages of IMM-H004 were shown in Figure 2B. 
pMCAO model was built on aged rats and IMM-H004 $(10 \mathrm{mg} / \mathrm{kg})$ was administrated at $6 \mathrm{~h}$ after ischemia, and rats were sacrificed $3 \mathrm{~h}$ after drug administration in all the following experiments. IMM-H004 significantly reduced the brain infarction and neurological dysfunction in rats with dosages of 5,10 , and $20 \mathrm{mg} / \mathrm{kg}$ compared with the rats received saline (Figure $2 \mathrm{~A}$ ). These results showed that the therapy dosage window of IMM-H004 is 5 to $20 \mathrm{mg} / \mathrm{kg}$ (Figure 2B,C).

A

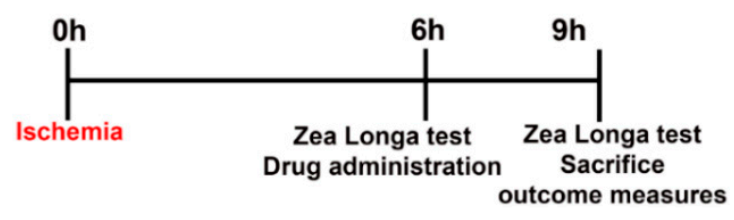

B

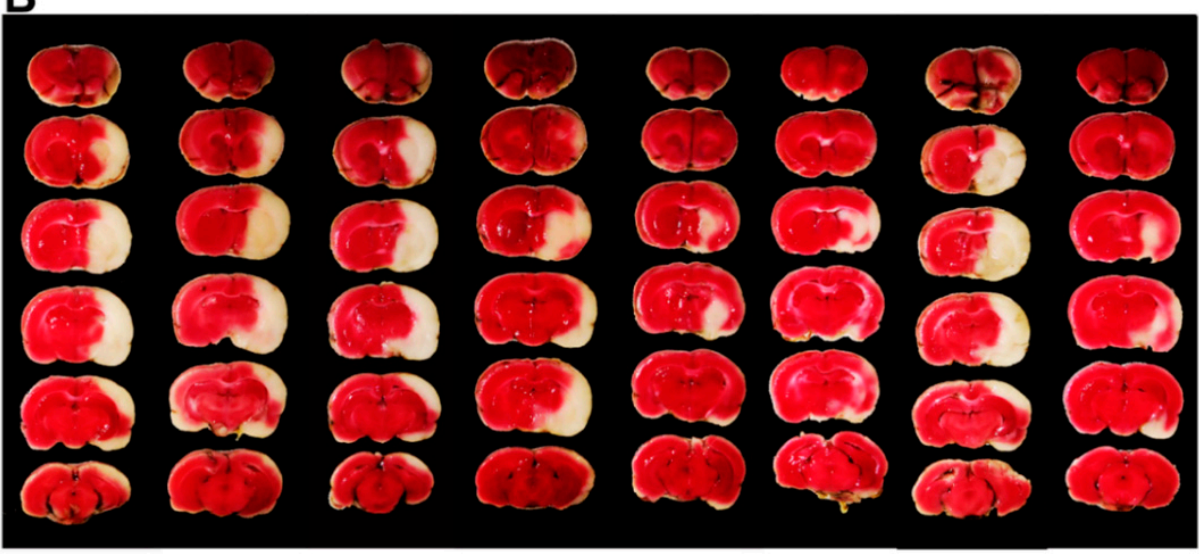

Model

1.25

2.5

5

10

20

EDA

URO

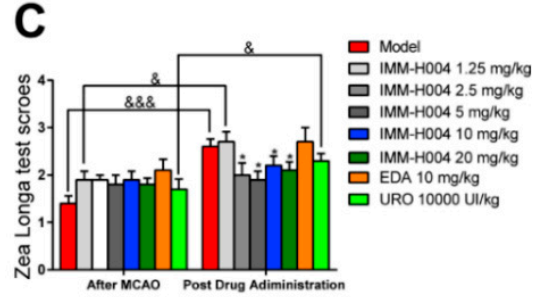

IMM-H004 (mg/kg)
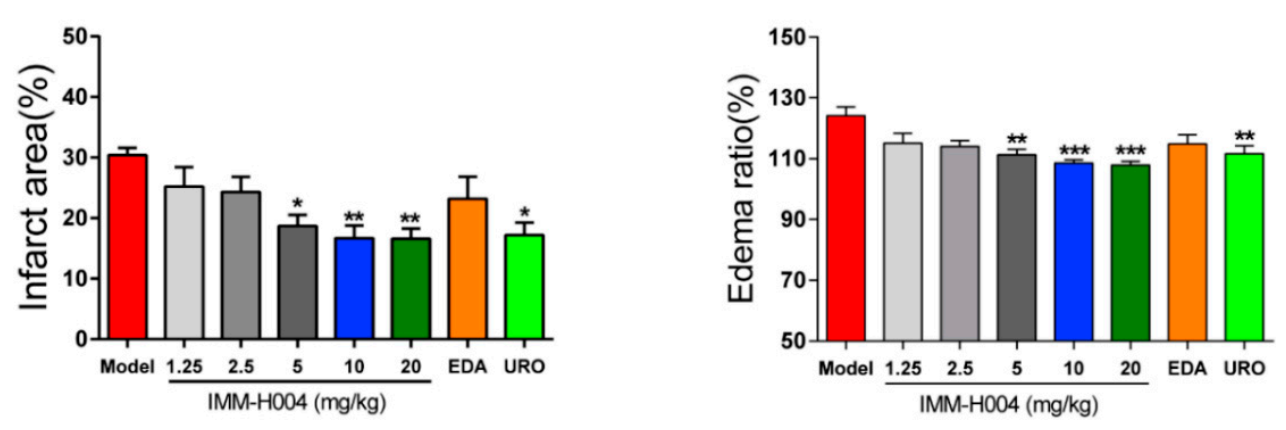

Figure 2. Therapeutic dosage window experiment of IMM-H004. (A) The timeline diagram of theraputic dosage window experiment. (B) Representative images of TTC staining, and analysis of infarction area and edema ratio. (C) Statistical analysis of Zea Longa test scores. Data are shown as the mean \pm SD ( $n=10$ /group). ${ }^{*} p<0.05$ vs. model; ${ }^{* *} p<0.01$ vs. model; ${ }^{* * *} p<0.001$ vs. model; $\& p<0.05$ vs. after $\mathrm{MCAO} ; \& \& \& p<0.001$ vs. after MCAO.

To investigate whether IMM-H004 has protective effects beyond $6 \mathrm{~h}$ after ischemia, we performed continuous administration of IMM-H004 1 time daily for 3 days. The first administration was at $6 \mathrm{~h}$ after $\mathrm{PMCAO}$, and we defined this time point as $0 \mathrm{~h}$, and the second and third administrations were at 24 and $48 \mathrm{~h}$. The survival rate and behavioral scores were counted at 0, 24, 48, and $72 \mathrm{~h}$ (Figure 3A). Rats were randomly assigned to four groups: 1 , pMCAO insult; 2 , pMCAO combined with IMM-H004 $(10 \mathrm{mg} / \mathrm{kg}) ; 3$, pMCAO combined with edaravone $(10 \mathrm{mg} / \mathrm{kg}) ; 4$, pMCAO combined with urokinase $(10,000 \mathrm{UI} / \mathrm{kg})$. All of the rats were sacrificed at $72 \mathrm{~h}$. As shown in Figure 3B-D, the $72 \mathrm{~h}$ survival rate of IMM-H004 treatment was better than urokinase treatment and the same as edaravone treatment. 
IMM-H004 and urokinase treatment can significantly improve the neurological deficits compared with that of the model group. TTC staining showed no significant difference among each group at $72 \mathrm{~h}$ after ischemia. These results demonstrated that continuous administration of IMM-H004 (10 mg/kg) showed improved survival compared to edaravone and urokinase after permanent focal cerebral ischemia in rats, however, the infarct size remains unchanged (Figure 3B-D).
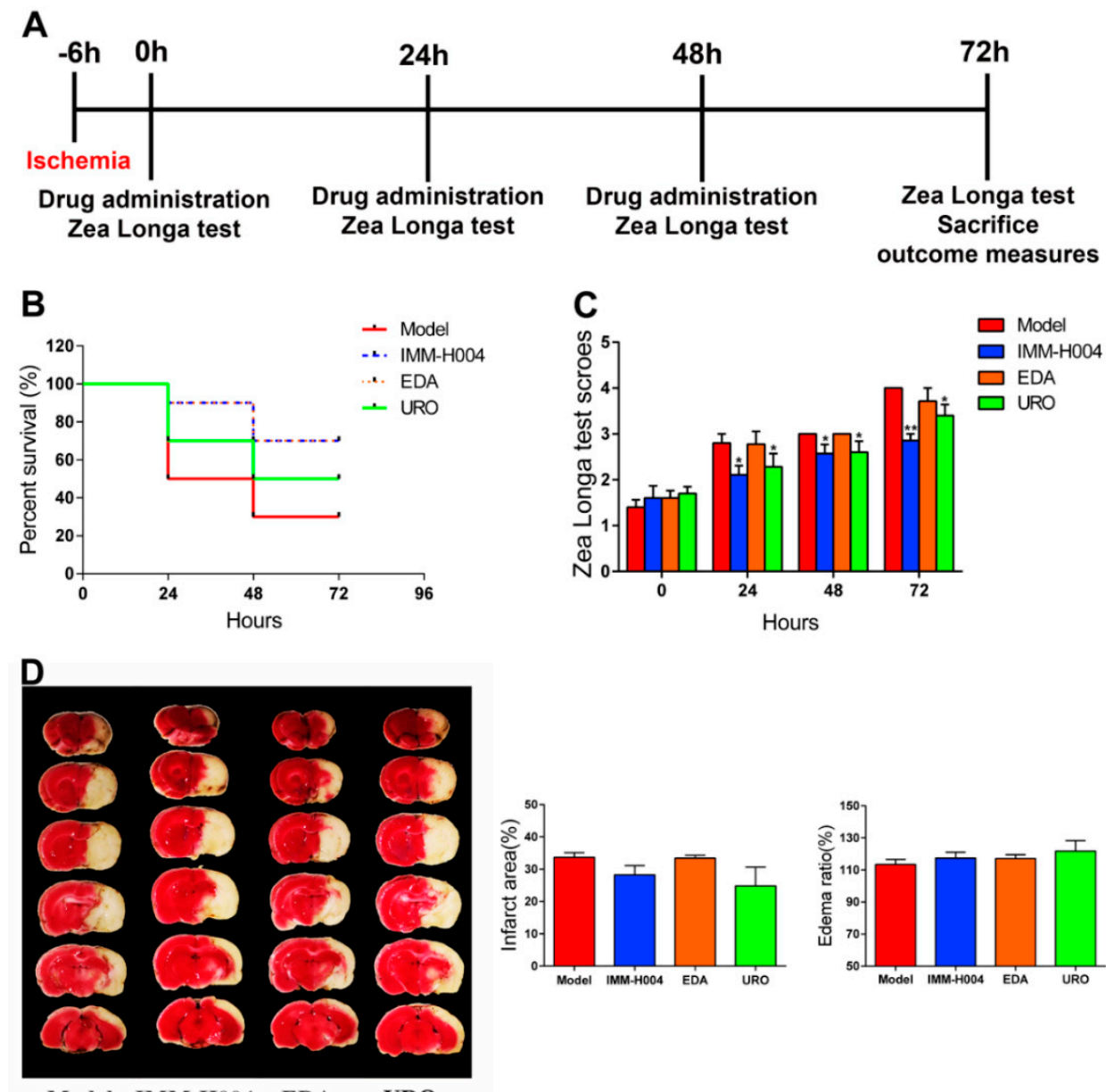

Model IMM-H004 EDA URO
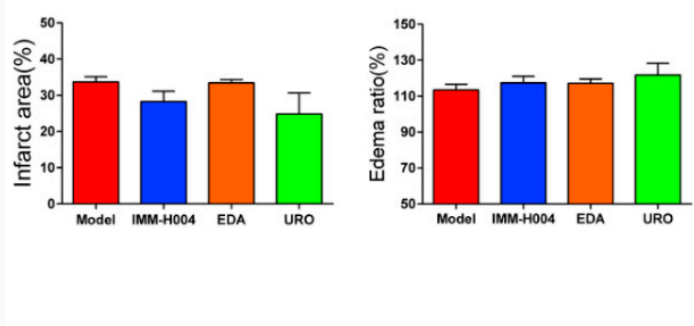

Figure 3. Successive administration of IMM-H004 once daily for three days protects against Permanent focal cerebral ischemia-induced brain injury in adult rats. (A) The timeline diagram of successive administration of IMM-H004 once daily for three days. (B) Statistical analysis of the $72 \mathrm{~h}$ survival rate. (C) Statistical analysis of Zea Longa test scores. (D) Representative images of TTC staining, and analysis of infarction area and edema ratio. Data are shown as the mean $\pm \operatorname{SD}\left(n=10\right.$ /group). ${ }^{*} p<0.05$ vs. model; ${ }^{* *} p<0.01$ vs. model.

Moreover, since clinical application for stroke therapy are frequently multiple drug administration daily, effects of IMM-H004 by repeated administration in one day was also studied. Drugs were administered at 3,6, and $12 \mathrm{~h}$ after ischemia $(0 \mathrm{~h})$. MRI and behavior tests were performed at $0,3,6,12$, and $24 \mathrm{~h}$ after ischemia (Figure $4 \mathrm{~A})$. The results showed that IMM-H004 $(10 \mathrm{mg} / \mathrm{kg}) \mathrm{multiple}$ dosing administration also pronouncedly reduced the brain infarct size and attenuated neurological deficits (Figure 4B,C). 

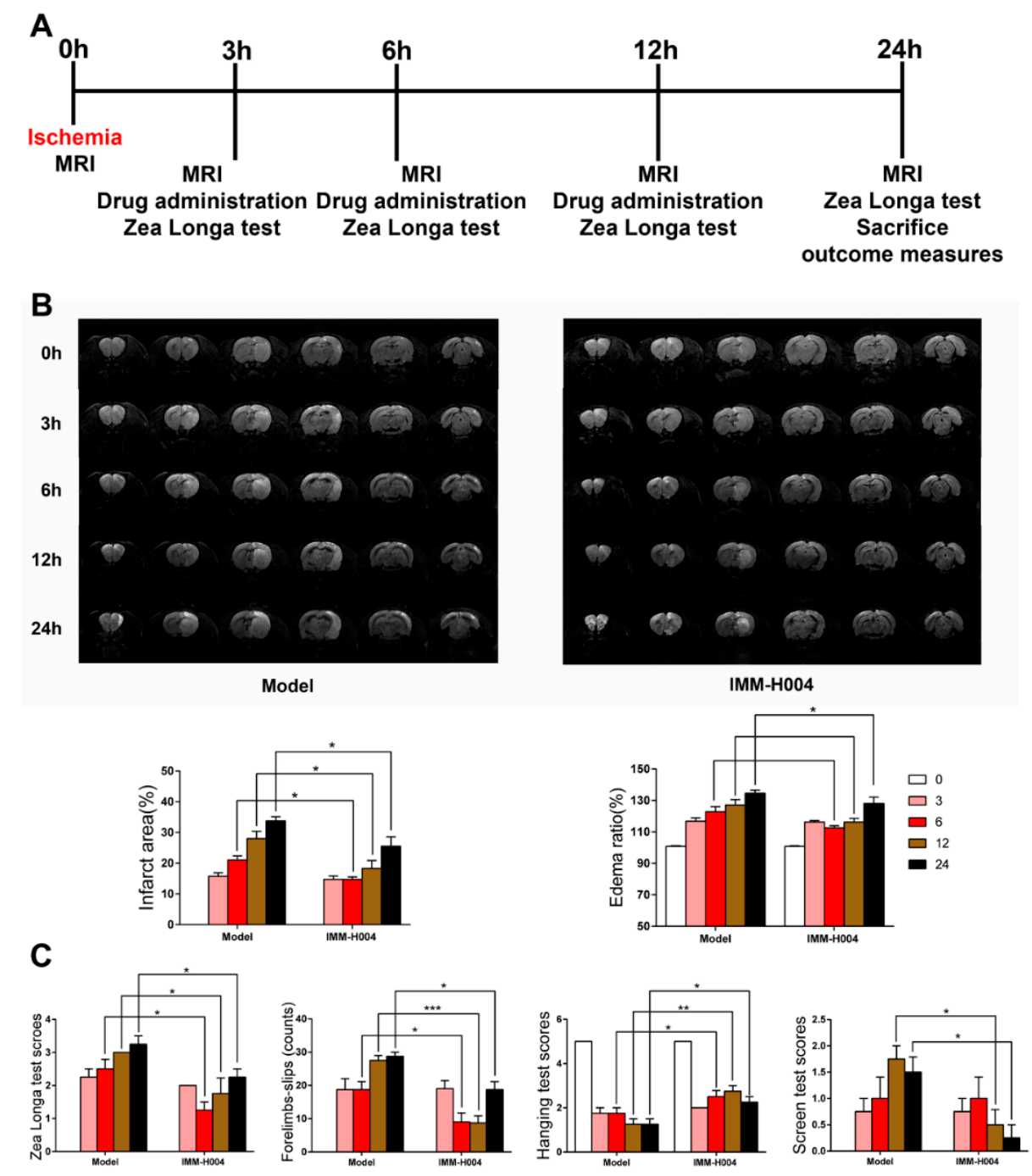

Figure 4. Successive administration of IMM-H004 3 times a day for one day protects against Permanent focal cerebral ischemia-induced brain injury in adult rats. (A) The timeline diagram of successive administration of IMM-H004 3 times a day for one day. (B) Representative coronal DWI images, and analysis of infarction area and edema ratio. (C) Statistical analysis of the Zea Longa test scores, Forelimbs-slips, Hanging test scores, and screen test scores. Data are shown as the mean \pm SD ( $n=10$ /group). ${ }^{*} p<0.05$ vs. model; ${ }^{* *} p<0.01$ vs. model; ${ }^{* * *} p<0.001$ vs. model.

\subsection{Effects of IMM-H004 on Permanent Focal Cerebral Ischemia Induced Brain Injury in Aged Rats}

To investigate whether IMM-H004 has protective effects on aged rats, pMCAO model was built on aged rats and IMM-H004 $(10 \mathrm{mg} / \mathrm{kg})$ was administrated at $6 \mathrm{~h}$ after ischemia, and rats were sacrificed $3 \mathrm{~h}$ after drug administration in all the following experiments. A total of 45 rats were randomly assigned to three groups: 1 , sham group; 2 , pMCAO insult group; 3 , pMCAO combined with IMM-H004 $(10 \mathrm{mg} / \mathrm{kg})$ treatment group. MRI scanning images showed that IMM-H004 treatment pronouncedly reduced the brain infarct size and edema volume (Figure 5A).

Moreover, after permanent focal cerebral ischemia, the number of normal CA1 hippocampus neurons, cortex neurons, and striatum neurons of the pMCAO treatment group sharply decreased, whereas the relict neurons showed karyopyknosis, anachromasis, nucleoli disappeared and vacuolization as assayed by Nissl staining (Figure 5B). IMM-H004 significantly protected the number of neurons in these brain regions from the stroke-induced reduction (Figure $5 \mathrm{~B}$ ). 


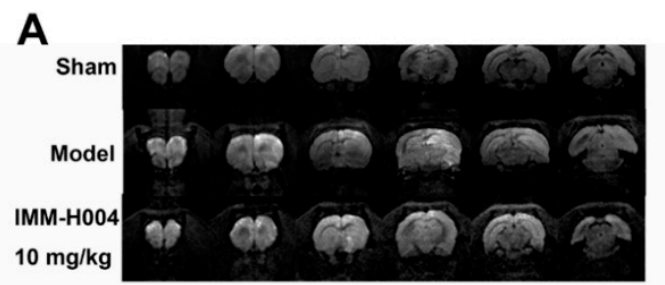

$6 \mathrm{~h}$

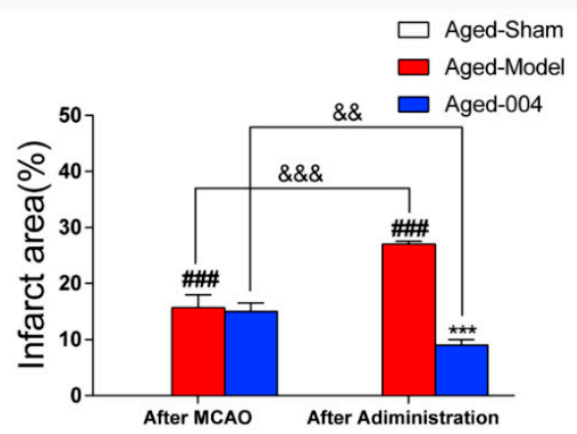

B

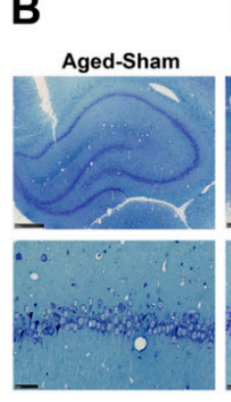

Hippocampus

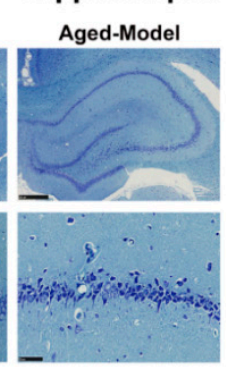

Cortex
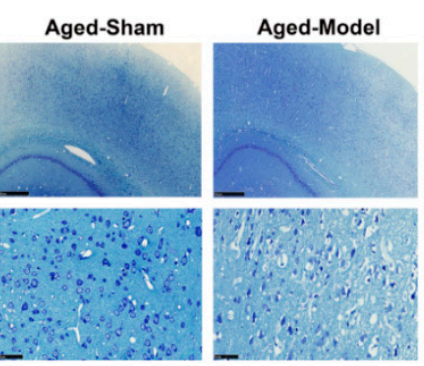

Striatum

Aged-Sham

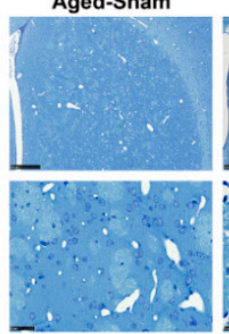

Aged-Model

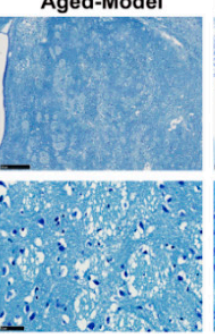

AgedIMM-H004 10mg/kg
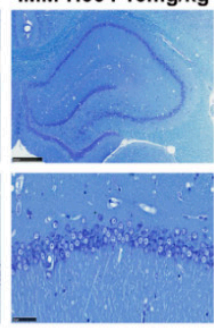

Aged-

IMM-H004 10mg/kg

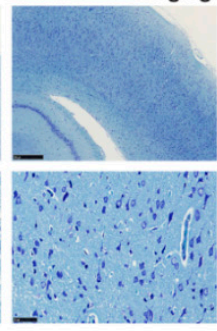

AgedIMM-H004 10mg/kg

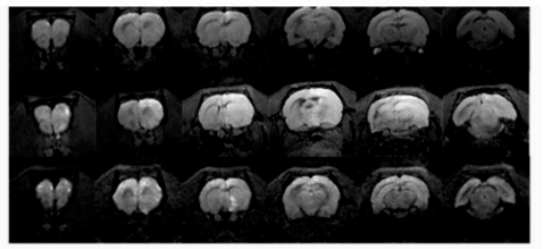

$9 \mathrm{~h}$
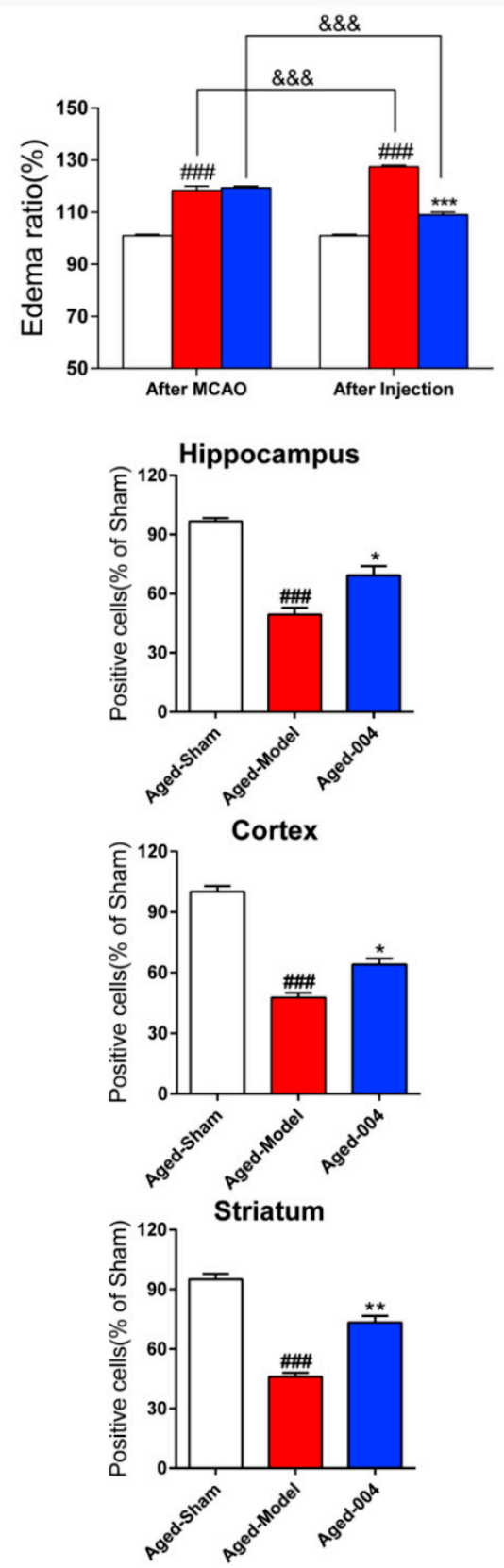

Figure 5. IMM-H004 protects against permanent focal cerebral ischemia-induced brain injury in aged rats. (A) Representative coronal DWI images, and analysis of infarction area and edema ratio. (B) Representative photographs of Nissl-stained hippocampal CA1, cortex, and striatum subfield from aged rats ( $50 \times$ and $400 \times$ magnification, scale bar: 500 and $50 \mu \mathrm{m})$ and positive cells ratio. All the data are shown as the mean $\pm \mathrm{SD}$; part of data are shown as the mean $\pm \mathrm{SD}$ after normalization to the sham ( $n=6$ /group). \#\#\# $p<0.001$ vs. sham; ${ }^{*} p<0.05$ vs. model; ${ }^{* *} p<0.01$ vs. model; ${ }^{* * *} p<0.001$ vs. model; $\& \& p<0.01$ vs. after MCAO; \&\&\& $p<0.001$ vs. after MCAO. 


\subsection{IMM-H004 Inhibits Brain Inflammation after Permanent Focal Cerebral Ischemia in Aged Rats}

To elucidate the anti-inflammation mechanism of IMM-H004 in permanent focal cerebral ischemia-induced brain injury of aged rats, the expression of CKLF1 in hippocampus, cortex, and striatum of brain were assayed by immunohistological staining. The expression of CKLF1 was low in the sham group, however, CKLF1 was increased significantly in pMCAO treatment group, and treatment with IMM-H004 (10 mg/kg) significantly decreased the expression of CKLF1 (Figure 6). The expression of CCR4 was detected by immunofluorescence staining. The results showed that the expression levels of CCR4 in the hippocampus, cortex, and striatum of aged rat have no significant change among all the groups (Figure 7A).
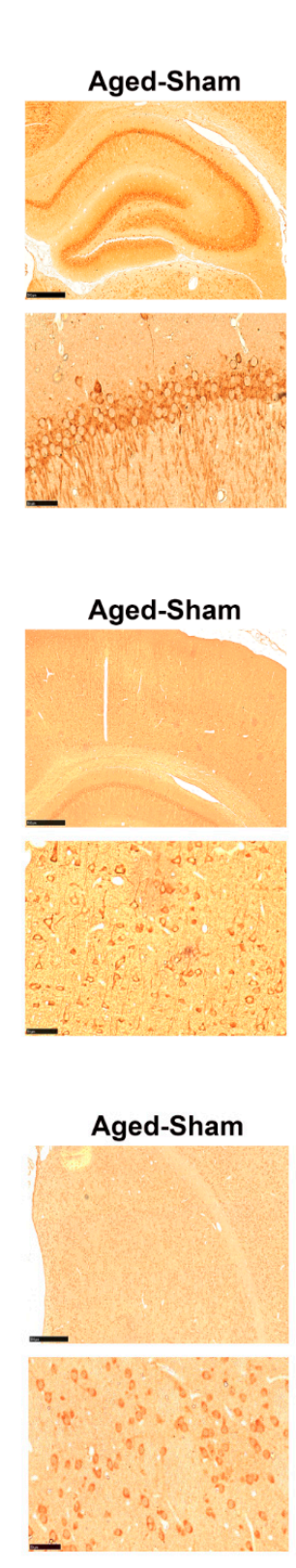

\section{Hippocampus}

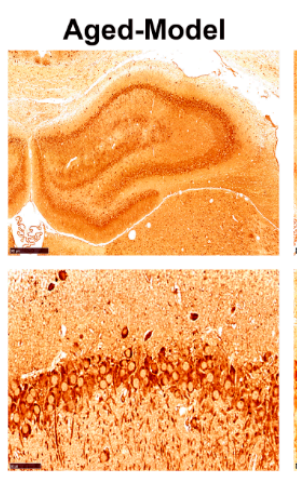

Cortex

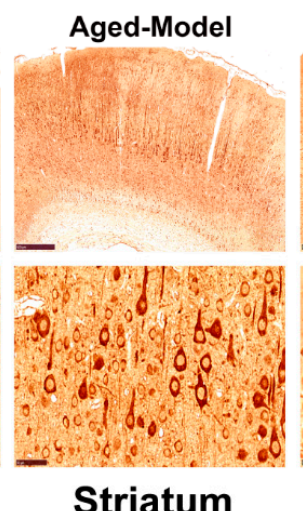

iatum

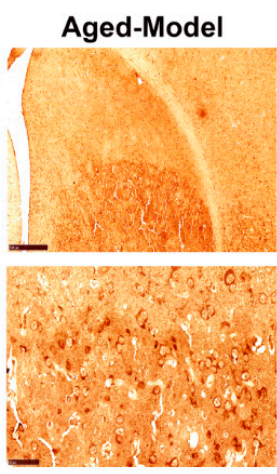

AgedIMM-H004 10mg/kg

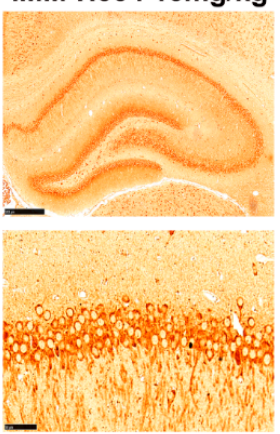

Aged-

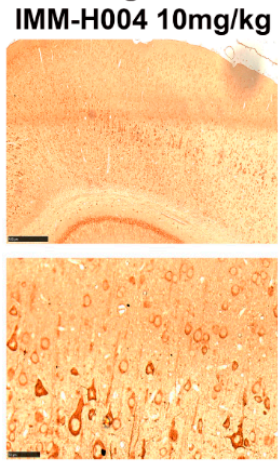

AgedIMM-H004 10mg/kg

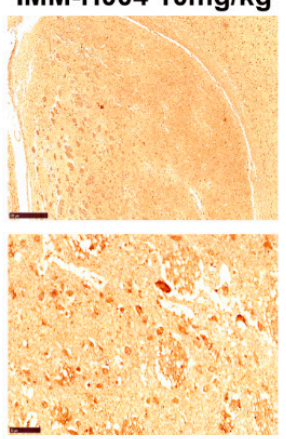

Hippocampus

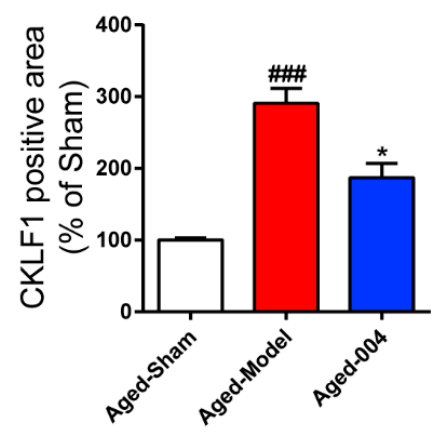

Cortex

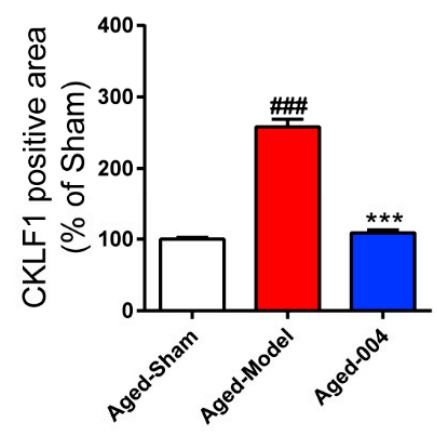

Striatum

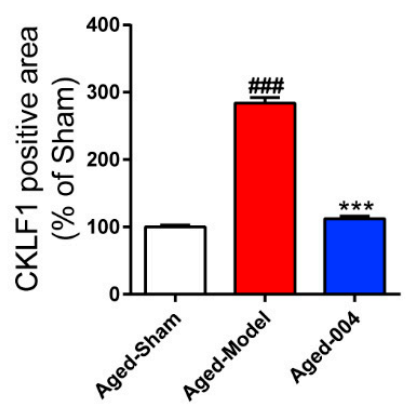

Figure 6. IMM-H004 decreases the expression of CKLF1 in ischemic brain. Expression of CKLF1 in hippocampal CA1, cortex, and striatum subfield from aged rats by immunohistochemical staining (50 $\times$ and $400 \times$ magnification, scale bar: 500 and $50 \mu \mathrm{m}$ ). All the data are shown as the mean \pm SD; part of data are shown as the mean $\pm \mathrm{SD}$ after normalization to the sham ( $n=6 /$ group). \#\#\# $p<0.001$ vs. sham; ${ }^{*} p<0.05$ vs. model; ${ }^{* * *} p<0.001$ vs. model. 
A Hippocampus
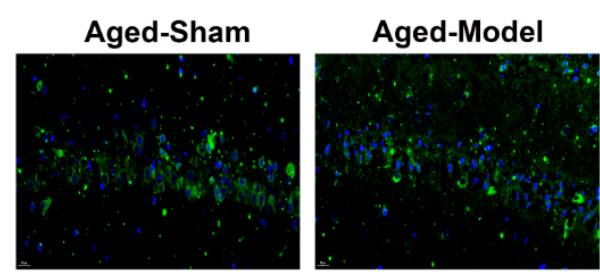

Cortex

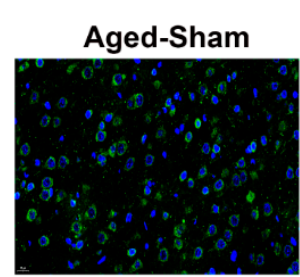

Aged-Model

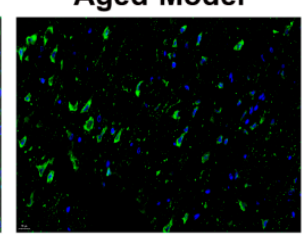

Striatum
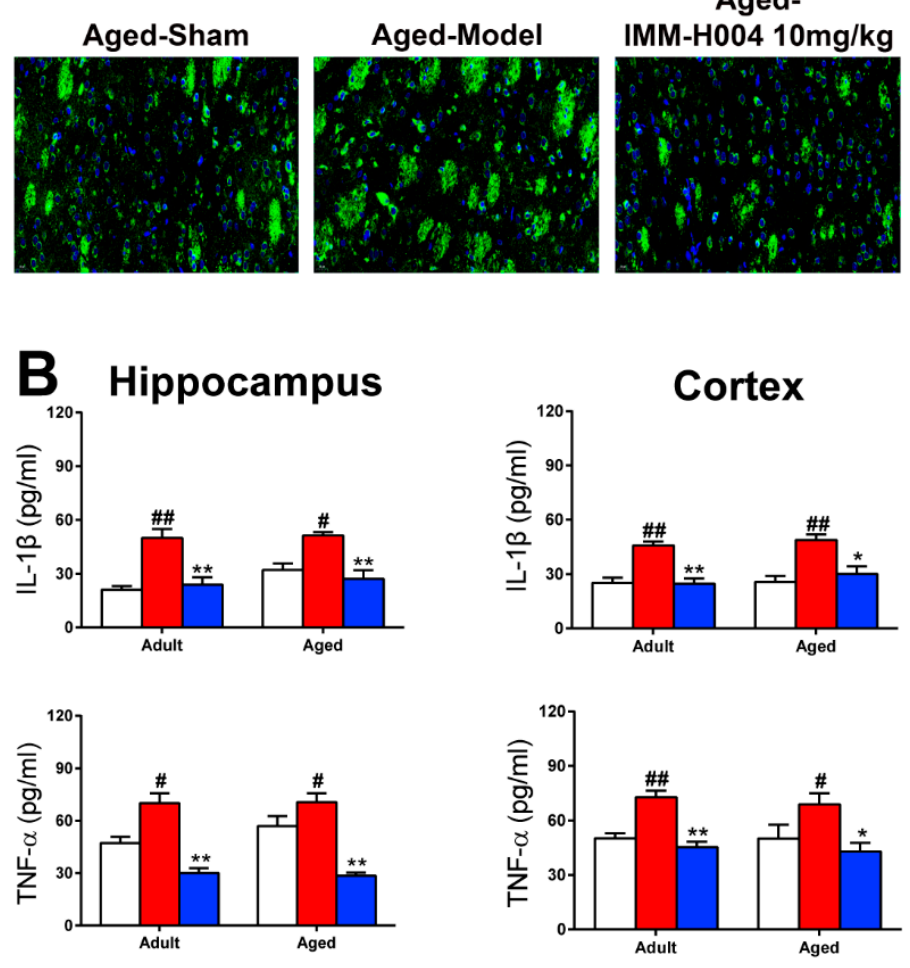
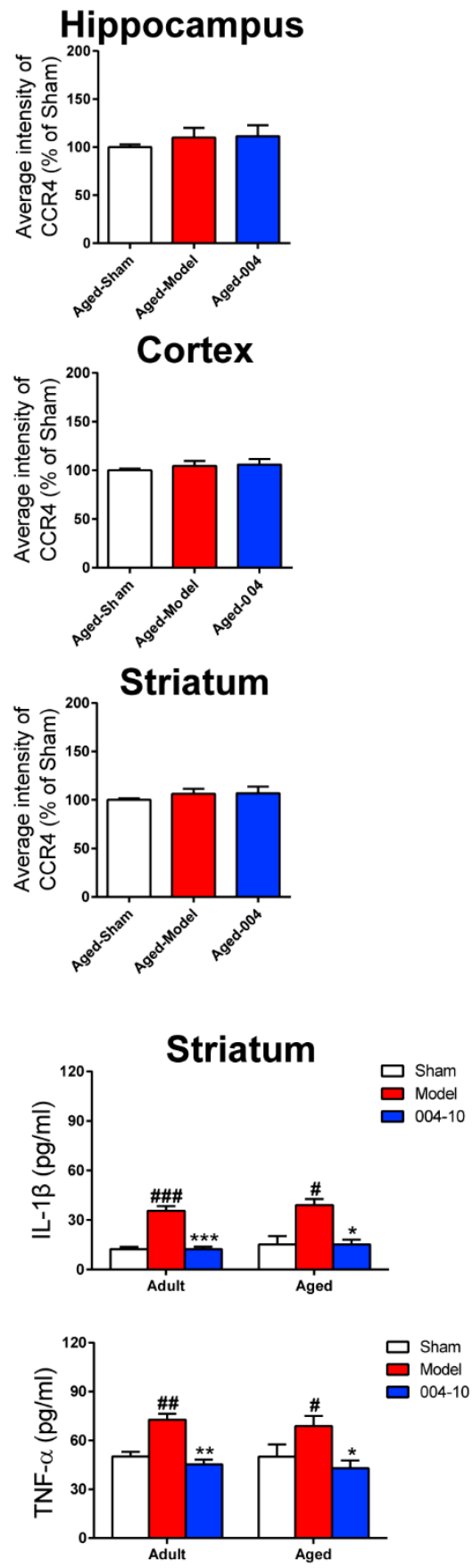

Figure 7. IMM-H004 dereases the inflammatory response in brain of aged rats. (A) Expression of CCR4 in hippocampal CA1, cortex, and striatum subfield from aged rats by immunofluorescence staining ( $400 \times$ magnification, scale bar: $50 \mu \mathrm{m}$ ). (B) Effect of IMM-H004 on IL-1 $\beta$ and TNF- $\alpha$ levels in hippocampus, cortex, and striatum of aged rats. All the data are shown as the mean $\pm \mathrm{SD}$; part of data are shown as the mean $\pm \mathrm{SD}$ after normalization to the sham ( $n=6 /$ group). $\# p<0.05 \mathrm{vs}$. sham; \#\# $p<0.01$ vs. sham; \#\#\# $p<0.001$ vs. sham; ${ }^{*} p<0.05$ vs. model; ${ }^{* *} p<0.01$ vs. model; ${ }^{* * *} p<0.001$ vs. model.

Inflammatory cytokines in the hippocampus, cortex, and striatum of aged rats were assayed by ELISA to determine the anti-inflammation effects of IMM-H004. The expression of IL- $1 \beta$ and TNF- $\alpha$ in hippocampus, cortex, and striatum of aged rats were all markedly increased in response to pMCAO operation, while these cytokines were significantly reduced by treatment with IMM-H004 $(10 \mathrm{mg} / \mathrm{kg})$. Similar effects were shown on adult rats (Figure 7B). 
As assayed by qPCR, the mRNA expression levels of CKLF1, IL-1 $\beta$, and TNF- $\alpha$ in the hippocampus, cortex, and striatum of aged rats were markedly increased by pMCAO treatment and that the expression levels were decreased in the IMM-H004 $(10 \mathrm{mg} / \mathrm{kg})$ treatment group, and equally, the expression levels of CCR4 showed no differences among all of the groups (Figure 8A). The protein expression of CKLF1, CCR4, p-NF-kB, NF- $\mathrm{BB}, \mathrm{IL}-1 \beta$, and TNF- $\alpha$ in the hippocampus, cortex, and striatum of brain were detected by western blotting. IMM-H004 $(10 \mathrm{mg} / \mathrm{kg})$ treatment can decrease the expression levels of CKLF1, p-NF- $\mathrm{BB}, \mathrm{IL}-1 \beta$, and TNF- $\alpha$, which were increased by pMCAO treatment, whereas the expression levels of CCR4 showed no differences among all of the groups (Figure 8B).
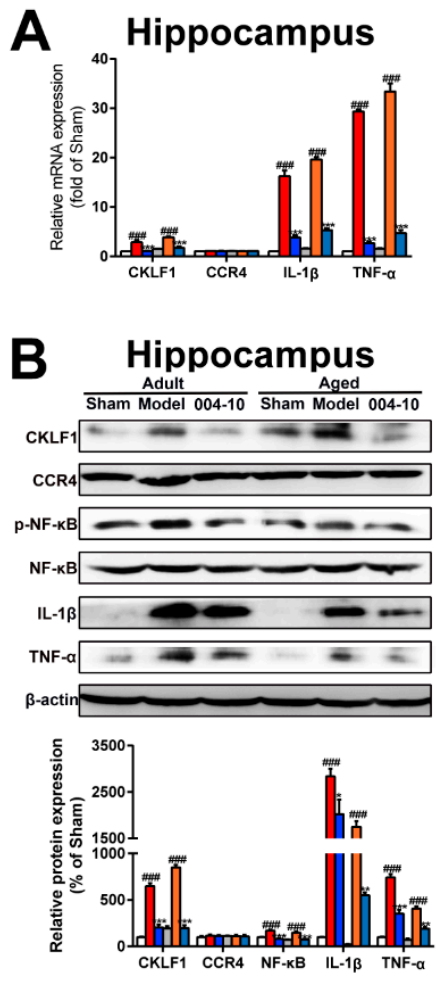

Cortex

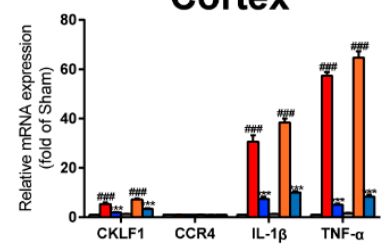

Cortex
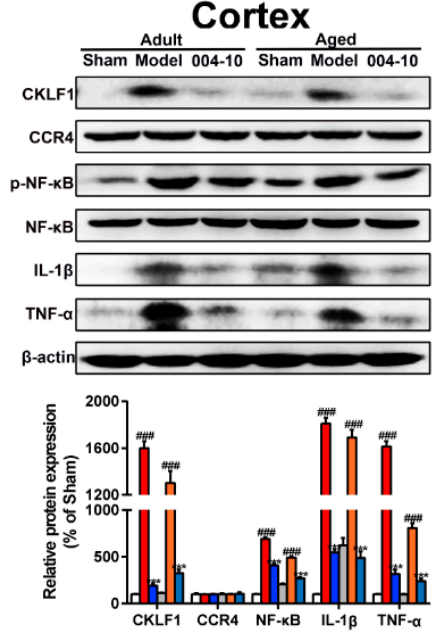

Striatum

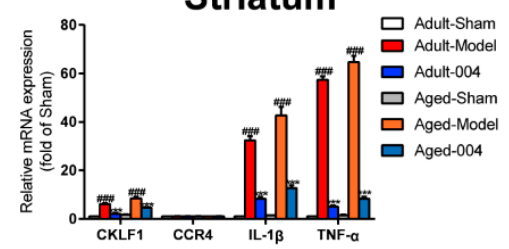

Striatum

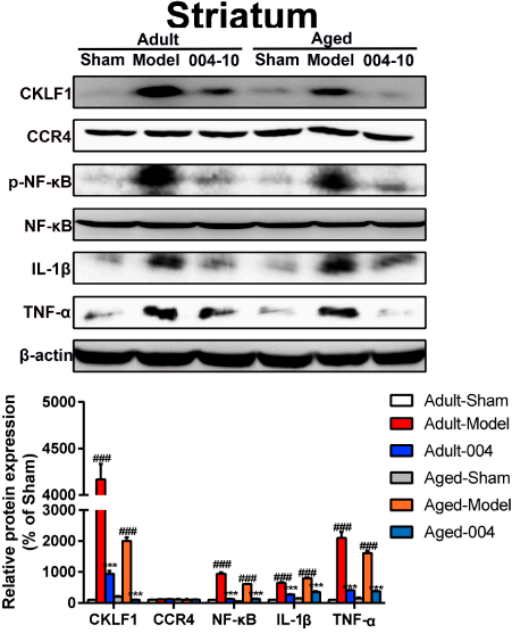

Figure 8. IMM-H004 decreases Permanent focal cerebral ischemia-induced brain inflammation in aged rats. (A) RT-PCR analysis for mRNA levels of CKLF1, CCR4, IL-1 $\beta$, and TNF- $\alpha$ in hippocampus, cortex, and striatum of aged rats. (B) Representative blots and densitometry data for CKLF1, CCR4, IL-1 $\beta$, and TNF- $\alpha$ hippocampus, cortex, and striatum of aged rats. Data are shown as the mean \pm SD after normalization to the sham ( $n=6$ /group). \#\#\# $p<0.001$ vs. sham; ${ }^{* *} p<0.01$ vs. model; ${ }^{* * *} p<0.001$ vs. model.

\subsection{Effects of IMM-H004 on Cardiopulmonary Complications in Aged Rats}

An overall view of the distribution of myocardial and lung damage of aged rats by HE staining was shown in Figure 9A. No obvious abnormalities were observed in the sham group, whereas myocardial damages were found in the pMCAO operated group characterized by eosinophilic changes in intensity, cytoplasmic vacuolization, and contraction band anomaly. IMM-H004 $(10 \mathrm{mg} / \mathrm{kg})$ treatment ameliorated the myocardial damages significantly. Similarly, no obvious abnormalities were observed in the lung of rats with sham operation, whereas remarkable lung damages were found in rats suffered with pMCAO operation, which characterized by inflammatory cell infiltration, alveolar wall thickening, pulmonary interstitial edema, and hemorrhage. Treatment with the IMM-H004 $(10 \mathrm{mg} / \mathrm{kg})$ could prevent lung damage markedly. LDH leakage in heart was also determined to evaluate the cardiac damage. As shown in Figure 9B, pMCAO operation induced a huge release of $\mathrm{LDH}$, and IMM-H004 $(10 \mathrm{mg} / \mathrm{kg})$ treatment could significantly inhibit the release of LDH. 
A
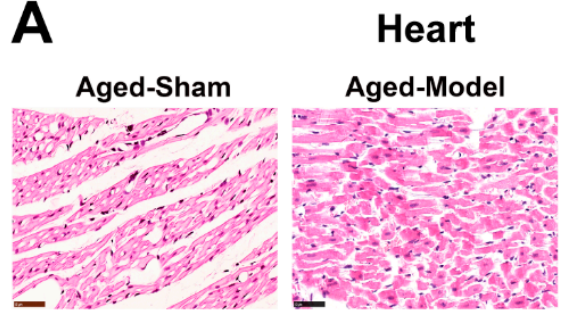

Lung

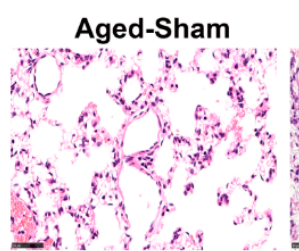

C
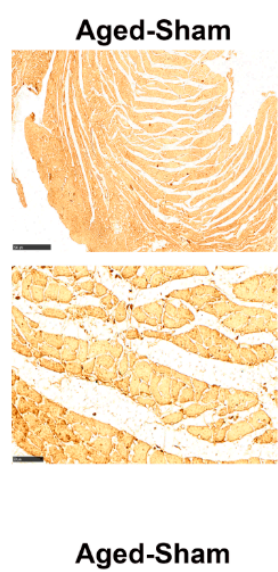

Aged-Model

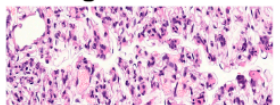

Heart

Aged-Model
Aged-

IMM-H004 10mg/kg

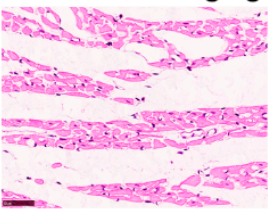

Aged-

IMM-H004 10mg/kg

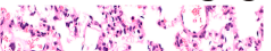

(1)

s.t.

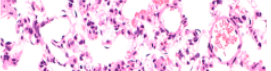

1.
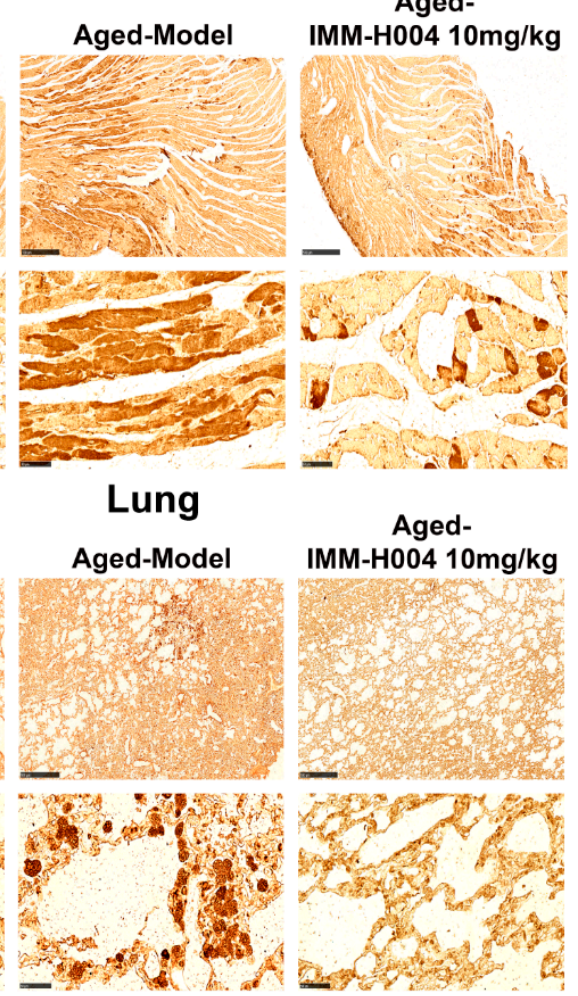

B

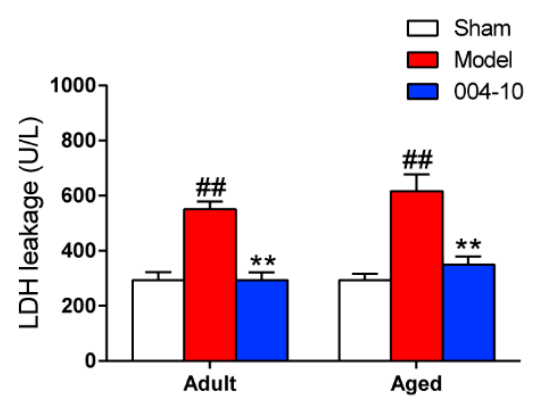

Heart

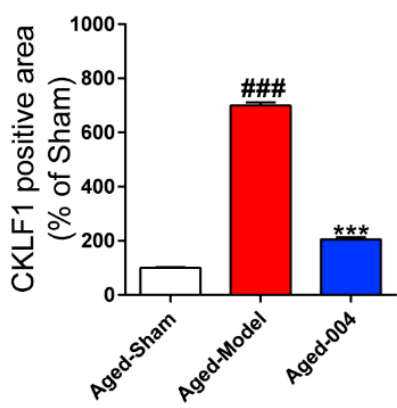

Lung

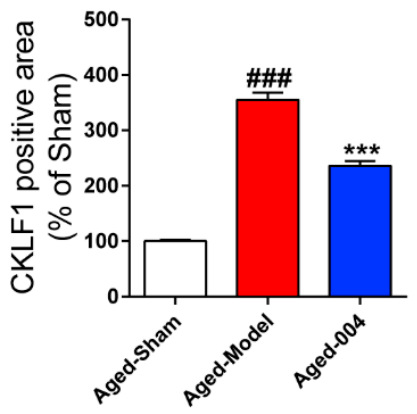

Figure 9. IMM-H004 protects against Permanent focal cerebral ischemia-induced cardiopulmonary complications in aged rats. (A) Representative photographs of HE-stained heart and lung subfield from aged rats $(400 \times$ magnification, scale bar: $50 \mu \mathrm{m})$. (B) Effect of IMM-H004 on LDH level in heart of aged rats using an LDH assay kit. (C) Expression of CKLF1 in heart and lung subfield from aged rats by immunohistochemical staining ( $50 \times$ and $400 \times$ magnification, scale bar: 500 and $50 \mu \mathrm{m})$. All the data are shown as the mean $\pm \mathrm{SD}$; part of data are shown as the mean $\pm \mathrm{SD}$ after normalization to the sham $\left(n=6\right.$ /group). \#\# $p<0.01$ vs. sham; \#\#\# $p<0.001$ vs. sham; ${ }^{* *} p<0.01$ vs. model; ${ }^{* * *} p<0.001$ vs. model.

\subsection{IMM-H004 Suppresses Inflammation in Heart and Lung Post-Stroke of Aged Rats}

To determine the changes of inflammatory response in heart and lung, the inflammatory cytokines were detected. We found that secreted IL-1 $\beta$ and TNF- $\alpha$ levels in heart and lung were all markedly 
increased after pMCAO operation, while these inflammatory cytokines reduced significantly by IMM-H004 administration (Figure 10B).

A
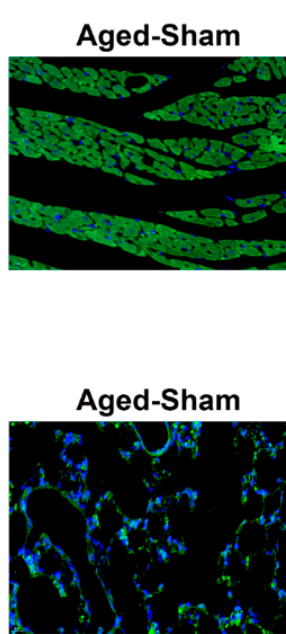

B
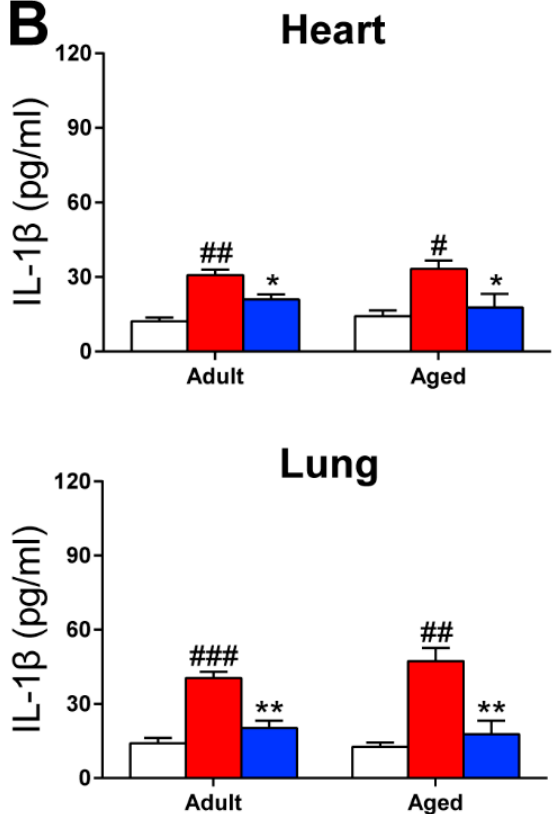

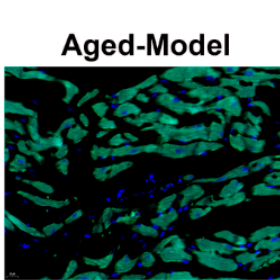

Lung

Aged-

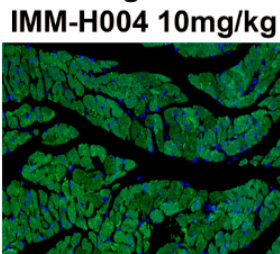

Aged-
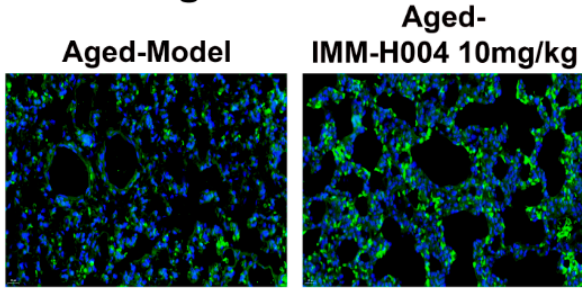

Heart

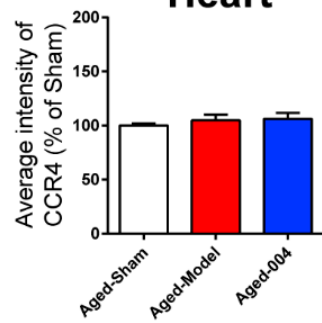

Lung

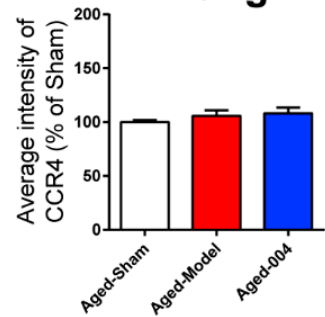

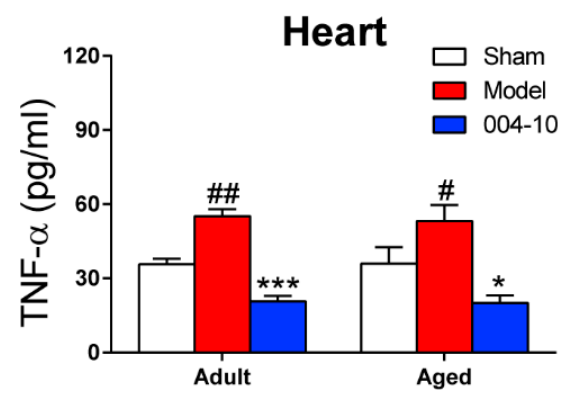

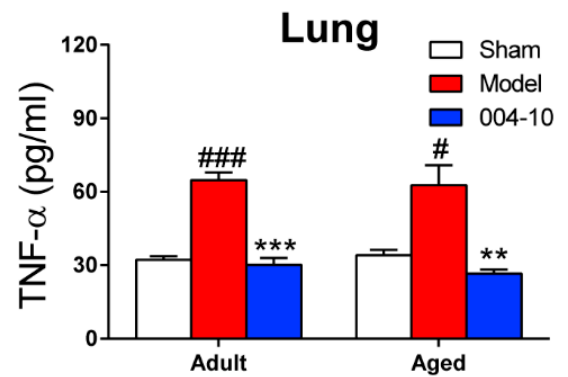

Figure 10. IMM-H004 decreases inflammation in heart and lung of aged rats. (A) Expression of CCR4 in heart and lung subfield from aged rats by immunofluorescence staining $(400 \times$ magnification, scale bar: $50 \mu \mathrm{m}$ ). (B) Effect of IMM-H004 on IL-1 $\beta$ and TNF- $\alpha$ levels in aged rats heart and lung using IL-1 $\beta$ and TNF- $\alpha$ assay kits. All the data are shown as the mean \pm SD; part of data are shown as the mean $\pm \mathrm{SD}$ after normalization to the sham ( $n=6$ /group). $\# p<0.05$ vs. sham; \#\# $p<0.01$ vs. sham; \#\#\# $p<0.001$ vs. sham; ${ }^{*} p<0.05$ vs. model; ${ }^{* *} p<0.01$ vs. model; ${ }^{* * *} p<0.001$ vs. model.

Then, the expression of CKLF1 was also determined in heart and lung by immunohistological staining. The results showed that expression of CKLF1 in heart and lung of the aged rats were both significantly increased after pMCAO operation. IMM-H004 treatment significantly decreased the expression of CKLF1 in heart and lung (Figure 9C). CCR4 expression of heart and lung (Figure 10A) showed neither significant changes after pMCAO operation, nor changed by treatment with IMM-H004 as assayed by immunofluorescence staining. The cardiopulmonary function detection including left ventricular systolic pressure (LVSP), left ventricular developed pressure (LVDP), the maximal 
ventricular pressure rise ratio during systolic period (+dp/dtmax), the maximal ventricular pressure decrease ratio during diastolic period (-dp/dtmax), minute ventilation (VE), and forced expiratory volume in the first second (FEV1) were determined in adult and aged rats. This was done at $9 \mathrm{~h}$ post-stroke ( $3 \mathrm{~h}$ post-drug). LVSP, $+\mathrm{dp} / \mathrm{dtmax},-\mathrm{dp} / \mathrm{dtmax}, \mathrm{VE}$, and FEV1 were markedly increased by pMCAO treatment and decreased by IMM-H004 while LVDP was opposite (Figure 11). As for the post-stroke infection, inflammation has been detected in the organ (Figure 10B). Because of the early complications of our detection, the observation time is $9 \mathrm{~h}$ after ischemia, and the infection may not be very serious. We will observe the relevant indicators in the further research. The mRNA and protein expression levels of CKLF1, CCR4, p-NF- $\mathrm{BB}, \mathrm{NF}-\mathrm{kB}$, IL-1 $\beta$, and TNF- $\alpha$ in heart and lung were determined. CKLF1, IL-1 $\beta$, p-NF- $\mathrm{kB}, \mathrm{NF}-\mathrm{kB}$, and TNF- $\alpha$ markedly increased by pMCAO treatment, and decreased by IMM-H004. The expression levels of CCR4 showed no differences among all of the groups (Figure 12A,B).
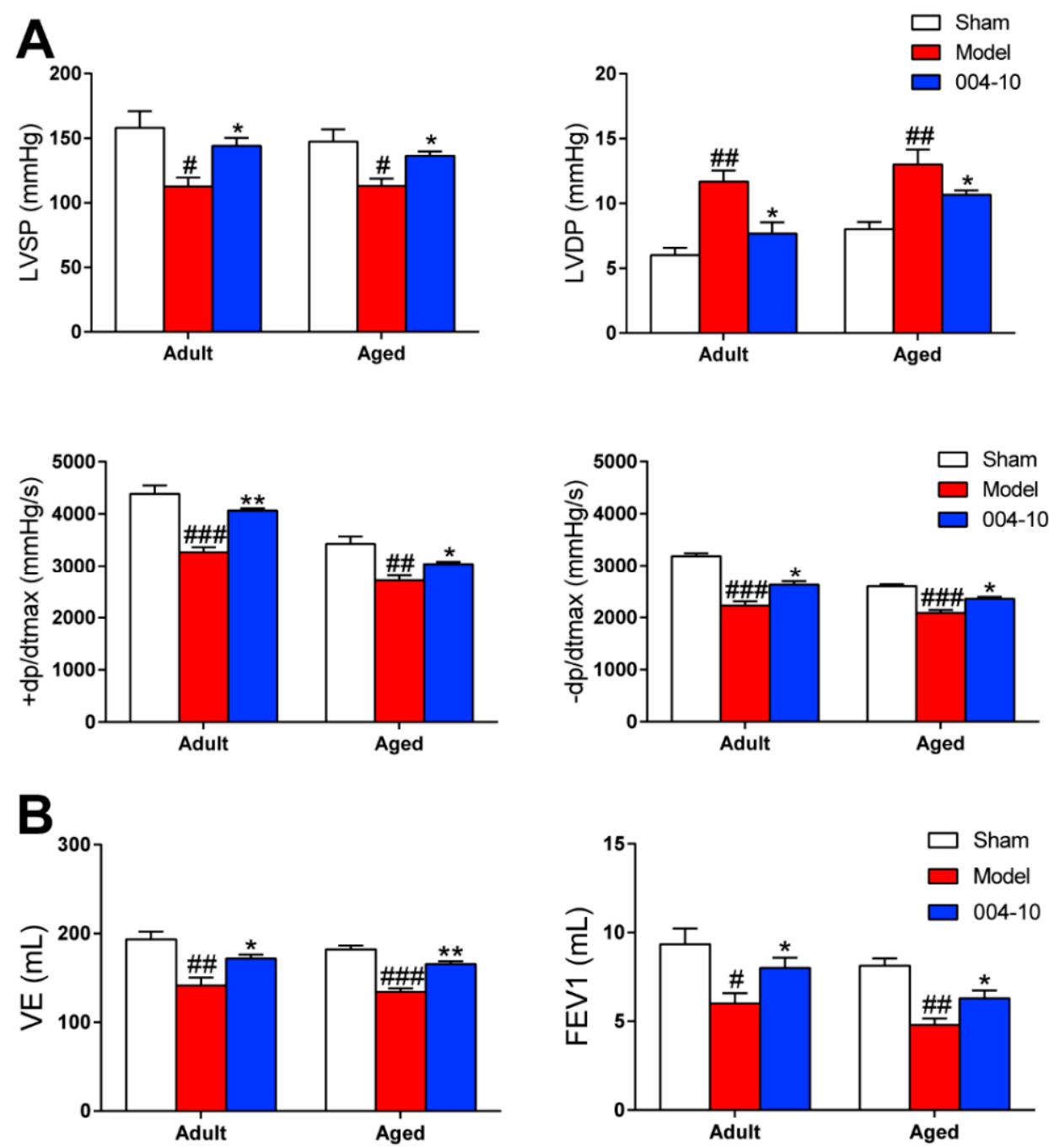

Figure 11. IMM-H004 protects cardiopulmonary function in adult and aged rats. (A) Effect of IMM-H004 on adult and aged rats cardiac function including the detection of LVSP, LVDP, $+\mathrm{dp} / \mathrm{dtmax}$, and -dp/dtmax. (B) Effect of IMM-H004 on adult and aged rats pulmonary function including the detection of VE and FEV1. All the data are shown as the mean $\pm \operatorname{SD}(n=6$ /group). $\# p<0.05$ vs. sham; \#\# $p<0.01$ vs. sham; \#\#\# $p<0.001$ vs. sham; ${ }^{*} p<0.05$ vs. model; ${ }^{* *} p<0.01$ vs. model. 

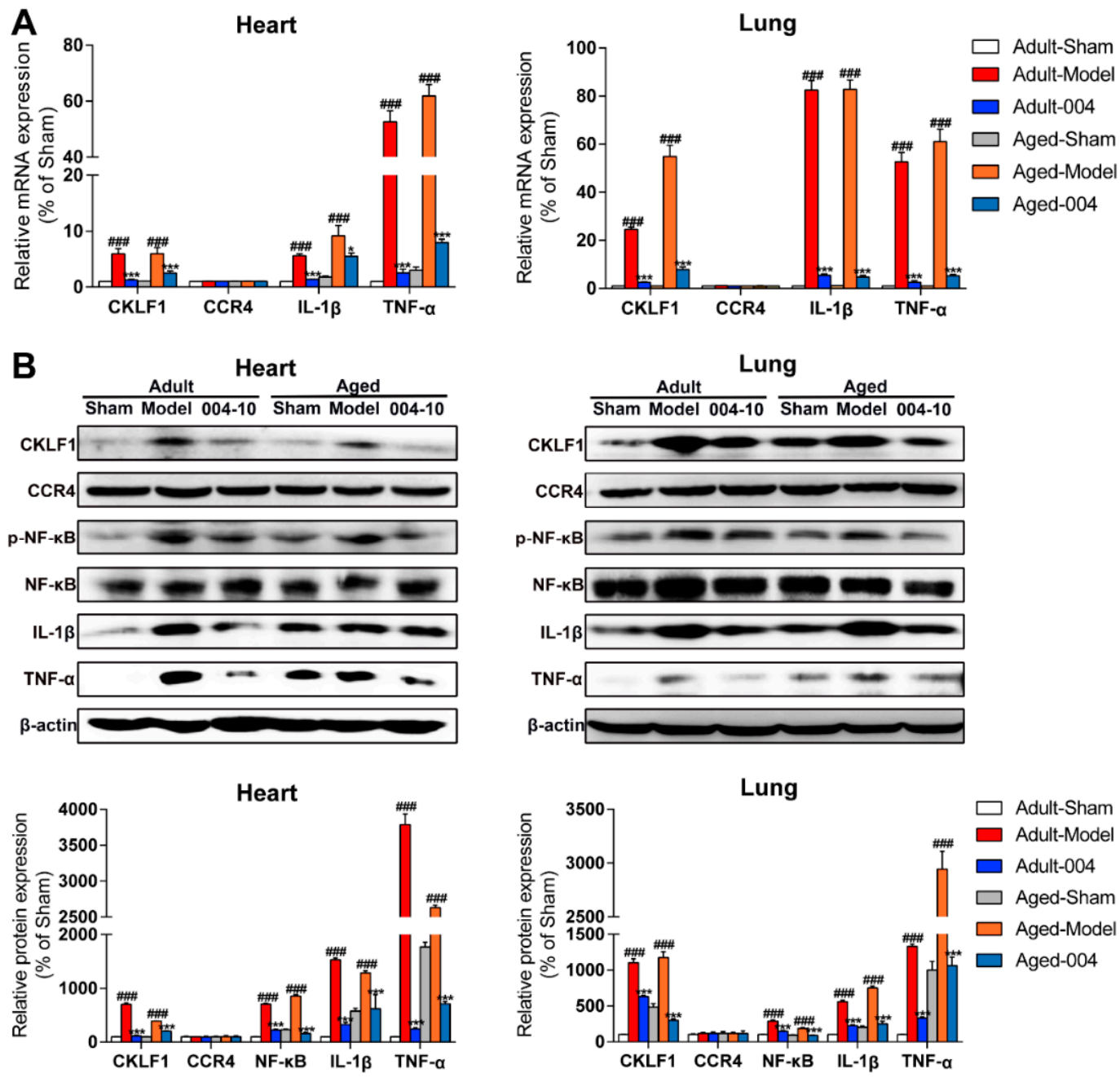

Figure 12. IMM-H004 decreases inflammation in heart and lung of aged rats. (A) Effect of IMM-H004 on aged rats heart and lung mRNA levels of CKLF1, CCR4, IL- $1 \beta$, and TNF- $\alpha$ as determined by quantitative RT-PCR. (B) Effect of IMM-H004 on the CKLF1, CCR4, IL-1 $\beta$, and TNF- $\alpha$ protein expression levels in aged rats heart and lung as assayed by Western blotting using a Gel-Pro analyzer (Media Cybernetics, Rockville, MD, USA). All the data are shown as the mean \pm SD; part of data are shown as the mean $\pm \mathrm{SD}$ after normalization to the sham $(n=6 /$ group $)$. \#\#\# $p<0.001$ vs. sham; ${ }^{*} p<0.05$ vs. model; ${ }^{* * *} p<0.001$ vs. model.

\subsection{IMM-H004 Exerts Protective Effects in Ischemic Brain through CKLF1 Mediated Inflammatory Pathway}

To investigate whether CKLF1 is necessary for IMM-H004 to exert its potency and elucidate the protective mechanism of IMM-H004, CKLF1 ${ }^{-/-}$rats were used. As shown in Figure 12, the pMCAO insult induced brain infarction and neurological deficits in CKLF1 deficient rats, but IMM-H004 showed no protective effects against brain damage on CKLF1 ${ }^{-/-}$rats (Figure $\left.13 \mathrm{~A}, \mathrm{~B}\right)$. These results demonstrated that IMM-H004 had no neuroprotective effect when CKLF1 is loss, suggesting the efficacy of IMM-H004 for brain protection is directly through targeting the CKLF1 (Figure 13A,B).

p-NF- $\mathrm{kB}, \mathrm{NF}-\mathrm{kB}, \mathrm{IL}-1 \beta$, and TNF- $\alpha$ in brain of CKLF1 ${ }^{-1-}$ rats were markedly increased by pMCAO insult, but IMM-H004 induced reduction in these increased expression levels was abolished in CKLF1 $1^{-/-}$rats (Figure 14A,B). The mRNA and protein expression levels of CCR4 showed no differences among all of the groups (Figure 14A,B). These results demonstrated that the protective effects of IMM-H004 against ischemic stroke-induced brain injury and inflammation is through the CKLF1 pathway involved with NF- $\mathrm{kB}$. 
A
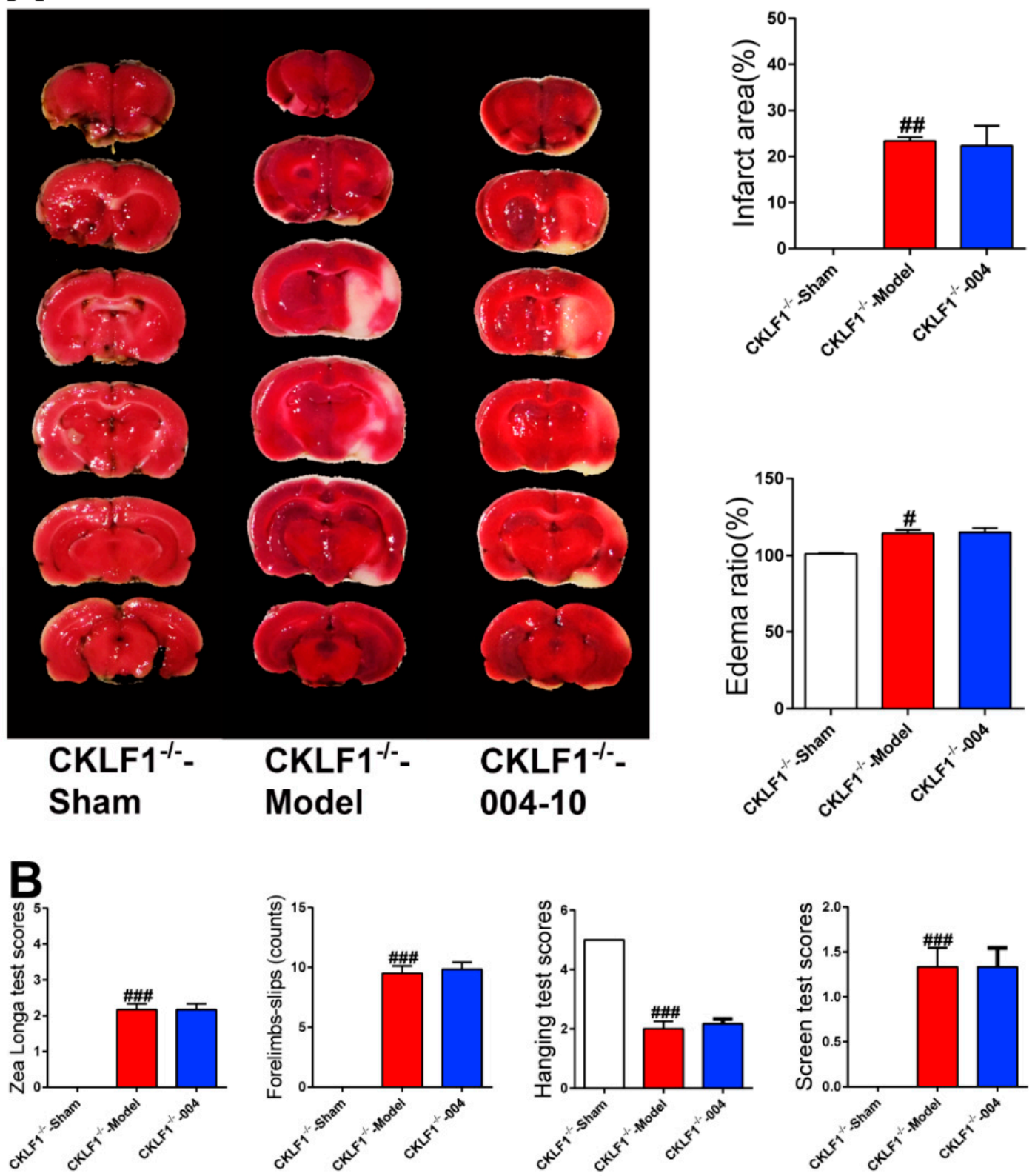

Figure 13. CKLF1 is necessary for IMM-H004 to exert protective effects against brain ischemia. (A) Representative TTC staining images, and analysis of infarction area and edema ratio. (B) Statistical analysis of the Zea Longa test scores, forelimb slips, hanging test scores, and screen test scores in $\mathrm{CKLF}^{-/-}$rats. All the data are shown as the mean $\pm \mathrm{SD}$, a part of data are shown as the mean $\pm \mathrm{SD}$ after normalization to the sham ( $n=6$ /group). \# $p<0.05$ vs. sham; \#\# $p<0.01$ vs. sham; \#\#\# $p<0.001$ vs. sham. 

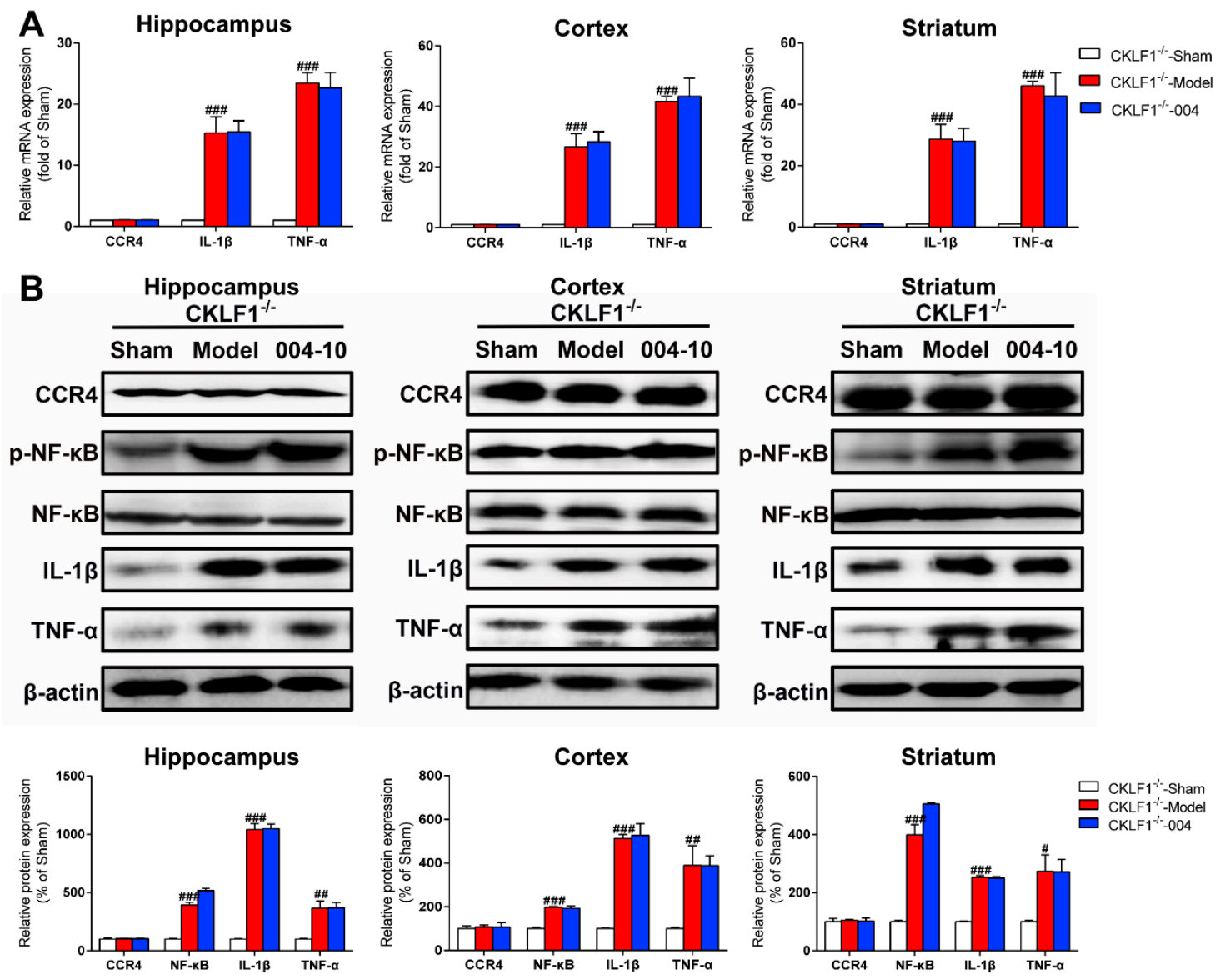

Figure 14. CKLF1 is necessary for IMM-H004 to anti-inflammation in ischemic brain. (A) RT-PCR analysis for Effect of IMM-H004 on expression levels of CCR4, IL-1 $\beta$, and TNF- $\alpha$ in the hippocampus, cortex, and striatum of $\mathrm{CKLF}^{-/-}$rats. (B) Western blot analysis for Effect of IMM-H004 on expression of CCR4, p-NF- $\mathrm{B}$, NF- $\mathrm{B}$, IL-1 $\beta$, and TNF- $\alpha$ in the hippocampus, cortex, and striatum of CKLF1 ${ }^{-/-}$ rats. All the data are shown as the mean $\pm \mathrm{SD}$, a part of data are shown as the mean $\pm \mathrm{SD}$ after normalization to the sham ( $n=6$ /group). \# $p<0.05$ vs. sham; \#\# $p<0.01$ vs. sham; \#\#\# $p<0.001$ vs. sham.

\subsection{IMM-H004 Protects against Ischemic Stroke Induced Cardiopulmonary Complications via CKLF1}

In $\mathrm{CKLF}^{-/-}$rats, the expression levels of $\mathrm{p}-\mathrm{NF}-\mathrm{kB}, \mathrm{NF}-\mathrm{kB}, \mathrm{IL}-1 \beta$, and TNF- $\alpha$ in heart and lung were all markedly increased by pMCAO treatment, but showed no decrease by IMM-H004 administration. Equally, the expression levels of CCR4 in mRNA and protein levels did not disturbed by pMCAO or treatment with IMM-H004 (Figure 15A,B). As verified by CKLF1 ${ }^{-/-}$ rats, the protective effects of IMM-H004 against permanent focal cerebral ischemia-induced cardiopulmonary complications is via the CKLF1 inflammation pathway involved with NF- $\mathrm{kB}$. 

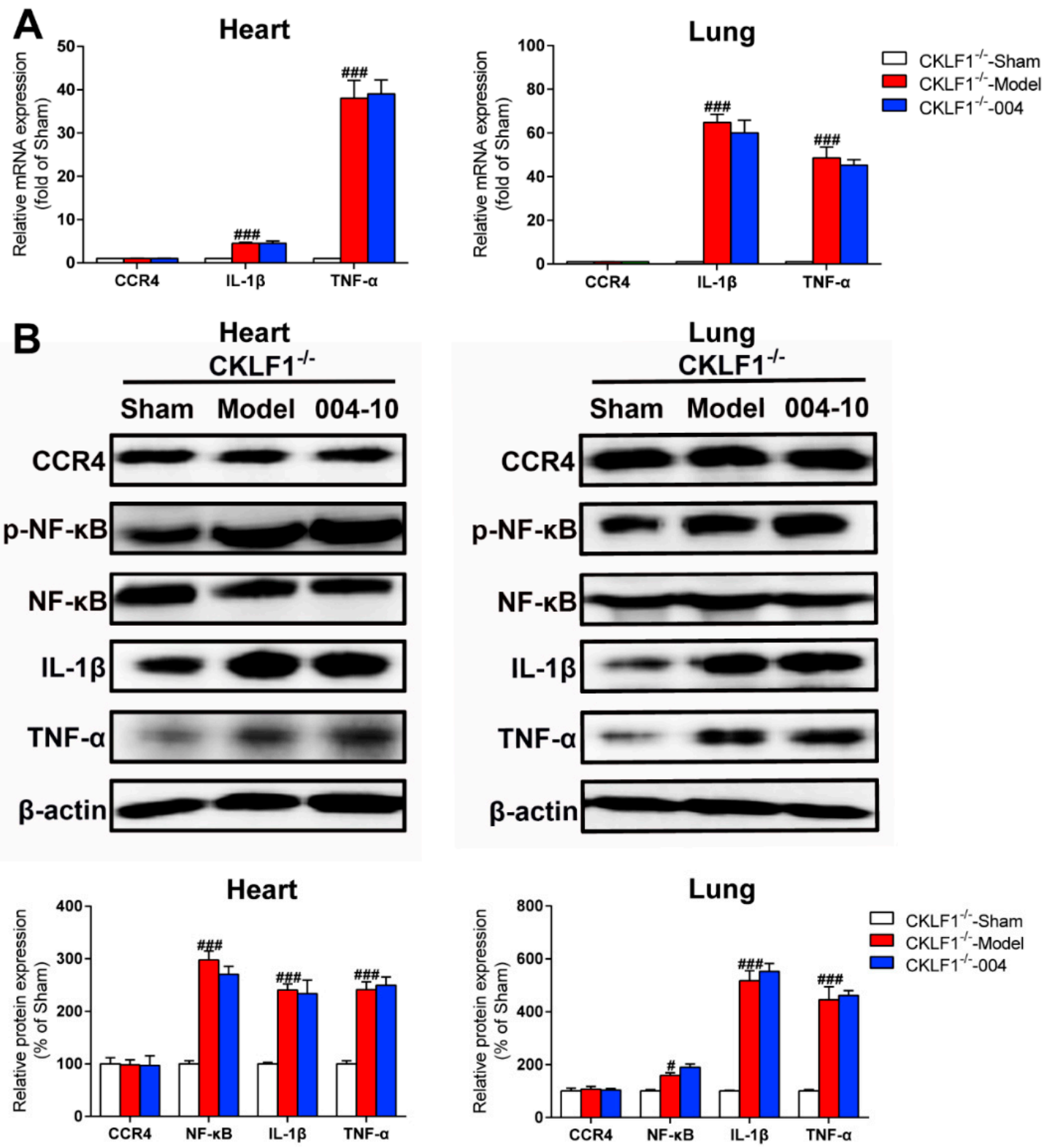

Figure 15. CKLF1 is necessary for IMM-H004 to suppress inflammation in heart and lung. (A) RT-PCR analysis for Effect of IMM-H004 on CCR4, p-NF-kB, NF-kB, IL-1 $\beta$, and TNF- $\alpha$ in the heart and lung of $\mathrm{CKLF}^{-/-}$rats. (B) Western blot analysis for the effect of IMM-H004 on CCR4, IL-1 $\beta$, and TNF- $\alpha$ in heart and lung of $\mathrm{CKLF} 1^{-/-}$rats. All the data are shown as the mean $\pm \mathrm{SD}$, a part of data are shown as the mean $\pm \mathrm{SD}$ after normalization to the sham ( $n=6 /$ group). \# $p<0.05$ vs. sham; \#\#\# $p<0.001$ vs. sham.

\section{Discussion}

Stroke remains a leading cause of death worldwide despite early revascularization therapies and progress in drug development. The main cause of death is stroke induced complications, manifested by heart and lung organ dysfunction. At present, no effective drugs are available for treatment of stroke and cardiopulmonary complications simultaneously. In present study, we found that IMM-H004 exerts protective effects on brain infarction and damage in heart and lung in a pMCAO rats model (Figures 1-12). Moreover, the therapeutic time window of IMM-H004 on permanent focal cerebral ischemia was determined to be $0-6 \mathrm{~h}$ which is wider than that of tPA. In order to extend the therapeutic time window, multiple drug administration daily was used. Figure 4 showed that 
IMM-H004 $(10 \mathrm{mg} / \mathrm{kg})$ multiple dosing administration also pronouncedly reduced the brain infarct size and attenuated neurological deficits. Because the protection mechanism of IMM-H004 is not thrombolytic therapy but anti-inflammation, so multiple dosing administration still have protective effect in long time beyond $6 \mathrm{~h}$. In the further research we will investigate the effects of multiple administrations for a longer period of time beyond $24 \mathrm{~h}$. Urokinase and edaravone are common clinical cerebral ischemia treatment drugs at present. Urokinase is a thrombolytic drug and edaravone is a strong antioxidant drug. Urokinase was chosen as the positive drug in this study for its therapeutic time window is $0-6 \mathrm{~h}$ same as the IMM-H004 [39,40]. We also chose edaravone as the positive drug which is widely used in ischemic stroke research [41-44].

The elderly are more prone to get ischemic stroke and vulnerable for stroke complications, we enrolled the aged rats in our study to investigate the efficacy of IMM-H004. Fortunately, IMM-H004 showed protective effects on both brain injury and cardiopulmonary complications in aged rats. These results suggested a potential for IMM-H004 to treat ischemic stroke in both adult and elderly. Additionally, IMM-H004 could not only protect the brain against damage, but also reduce the severity of cardiopulmonary complications. In clinical patients, stroke associated myocardial infarction and pneumonia mainly develop first weeks $[14,23]$. This work firstly reported that $9 \mathrm{~h}$ of ischemia could induce cardiopulmonary complications in aged rats, which is shorter than in human.

There are many theories for mechanisms of cerebral ischemia onset and progress, and inflammation is of particular interest at present [45]. IMM-H004 treatment could inhibit inflammatory response in brain of rats, as evidenced by the decrease of IL- $1 \beta$ and TNF- $\alpha$ in brain. Studies have shown that the cardiopulmonary complications after ischemic stroke is closely associated with inflammation [31,32]. We found that IMM-H004 inhibits the inflammation in heart and lung of ischemic rats. These results suggested that IMM-H004 exerts protective effects on brain injury and cardiopulmonary complications is partly through suppressing the inflammatory response after stroke.

IMM-H004 is a compound screened from a system of C27 (CKLF1 agonist peptide) and CCR4, and our previous studies have shown that CKLF1 may play an important and detrimental role in the early phase of a stroke [28-30,46,47]. Whether IMM-H004 is exert its protective effects through the CKLF1 is still unknown. In addition, whether CKLF1 is involved in the occurrence of cardiopulmonary complications is of our interest. In the present study, we found CKLF1 increased in ischemic brain and damaged heart and lung significantly after stroke, and IMM-H004 could decrease the expression of CKLF1 in these parts. As a receptor of CKLF1, CCR4 showed no obvious change among rats in sham, ischemia or IMM-H004 treatment groups. By the application of CKLF1 ${ }^{-/-}$rats, we confirmed that IMM-H004 shows protective effects is dependent on CKLF1. When CKLF1 is loss, its protective effects will also lose. Moreover, increased CKLF1 in heart and lung after ischemic stroke also suggested us CKLF1 may involve in the cardiopulmonary complications.

Immunosuppression plays a major role in cerebral ischemia injury induced complication, and is now more universally acknowledged to be the main explanation for susceptibility to infection after stroke $[48,49]$. The suppression of systemic immunity by the nervous system is thought to protect the brain from further inflammatory insult, yet this comes at the cost of increased susceptibility to infection after stroke. With regard to internal causes, stroke-mediated immunodeficiency increases the risk of infectious episodes, predominantly chest infection, within the first few days after stroke [50,51]. Immunodeficiency develops as early as $12 \mathrm{~h}$ after ischemic stroke and may persist for several weeks [52,53]; therefore, it plays a major role in causing pneumonia in post-stroke patients. Infection could trigger downstream inflammatory signaling cascades. A recent study indicates that experimental stroke models have indirectly revealed the pathologic effects of the ischemic brain on the heart. It suggests that inflammation factors are secreted by injured neuronal cells in the early stage of ischemic insult and subsequently reach the heart through the bloodstream in the later stages of stroke [32]. Inflammation is a key progress at the initiation of ischemia and post-stroke, involving a variety of immune cells and factors. Chemokines are a family of chemotactic cytokines that originally identified as factors attracting circulating leukocytes toward the site of inflammation or injury $[54,55]$. 
Chemokines generally play roles through interaction with their cognate receptors expressing on leukocytes and other cell type. As a chemokine, CKLF1 may involve in inflammatory response after stroke. In present study, we found that the increased CKLF1 after ischemic stroke is accompanied by excessive inflammation, both in the brain and periphery organs. IMM-H004 could both decrease the expression of CKLF1 and inflammatory cytokines. However, in CKLF1 ${ }^{-/-}$rats, IMM-H004 could not decrease the inflammatory cytokines anymore, which means the CKLF1 could mediate the followed inflammation partly. Interestingly, we found the CKLF1 $1^{-/-}$rats showed smaller infarction and lighter neurological deficient compared with the $\mathrm{CKLF} 1^{-/-}$rats. This finding indicated that CKLF1 plays a detrimental role after ischemia at least in our detected time points. NF- $\mathrm{KB}$ is considered the central transcription factor of inflammatory mediators [56,57]. Numerous studies have shown the cerebral ischemia will result in NF- $\mathrm{KB}$ activation [58,59]. In our present study, the same phenomenon was observed. NF-KB activated after ischemia, and IMM-H004 could inhibit the activation. In CKLF ${ }^{-/-}$ rats, although significant NF- $\kappa B$ activation is observed after cerebral ischemia, IMM-H004 showed no effect on NF- $\kappa B$ activation, which implies the CKLF1 mediated inflammation may be via the NF-kB pathway.

This study confirmed the protective effects of IMM-H004 on adult and aged rats, targeting brain injury and cardiopulmonary complications simultaneously. Moreover, IMM-H004 exerts its protective effects via inhibiting the CKLF1 mediated inflammation pathway, involving with NF- $\mathrm{KB}$. Despite the findings which have significance for the novel drug development in present study, it still has some limitations. Firstly, despite in clinical investigations or basic animal studies, females showed significant difference in incidence, mortality, and morbidity of stroke compared with males [60,61]. In the present study, we used only male subjects to minimize confounding variables, such as hormonal cycling and estropause for this first series of experiments. We are very aware of potential sex differences in stroke outcome [62] and in immune responses [63,64]. Our future research will enroll sex as a biological variable to clarify CKLF1 functions in ischemia stroke. Secondly, the upstream transcription factors of CKLF1 need to be determined. Thirdly, despite NF-KB, there are other pathways involved in the downstream influence inflammatory response. These problems need to be resolved in further studies to strengthen the theoretical foundation of IMM-H004 and promote its development.

\section{Materials and Methods}

\subsection{Experimental Animals}

All of the experimental protocols and animal care were performed according to the National Research Council's Guide for The Care and Use of Laboratory Animals. The project identification coed is 000353, which was approved by the Institutional Animal Care and Use Committee of the Peking Union Medical College and Chinese Academy of Medical Sciences in 10 September 2017. Male adult SD rats (6-8 weeks, 250-280 g) and male aged SD rats (26 months old, 500-600 g) were obtained from VITAL RIVER Laboratories, Beijing, China. CKLF1 ${ }^{-/-}$rats $(250-280 \mathrm{~g})$ were obtained from Key Laboratory of Human Disease Comparative Medicine, NHFPC, Institute of Laboratory Animal Science, Peking Union Medicine College, Chinese Academy of Medical Sciences, Beijing, China. All of the rats were housed under standard conditions. For the sample size calculation, preliminary experiment indicated that there was at least $10 \%$ difference in infarct volume between groups with standard deviation associated with these measurements to be 0.06 . Therefore, we required a minimum of six animals per group to detect such a difference at $95 \%$ confidence $(\alpha=0.05)$ and 0.8 power. The study was not pre-registered prior to examination of the data or observing the outcomes.

\subsection{Materials}

The compound IMM-H004 (molecular formula: $\mathrm{C}_{16} \mathrm{H}_{20} \mathrm{O}_{4} \mathrm{~N}_{2}$; molecular weight: 304; purity $>99 \%$ ) (Figure 1A) was provided by the Department of Chemosynthesis, Institute of Materia Medica, Chinese Academy of Medical Sciences and Peking Union Medical College (Beijing, China); 
all of the IMM-H004 in this paper is IMM-H004 citrate. Positive drugs: edaravone $\left(\mathrm{C}_{10} \mathrm{H}_{10} \mathrm{~N}_{2} \mathrm{O}\right)$ was purchased from Simcere (Nanjing, China), and urokinase $\left(\mathrm{C}_{21} \mathrm{H}_{25} \mathrm{BrN}_{2} \mathrm{O}_{3}\right)$ was purchased from the National Institutes for Food and Drug Control (Beijing, China). These compounds were dissolved in physiological saline for in vivo experiments.

\subsection{Rat $p M C A O$ Model and Drug Administration}

Permanent focal cerebral ischemia was induced by permanent middle cerebral artery occlusion (pMCAO) for $0,3,6,9,12,24$, or $72 \mathrm{~h}$, as described previously (Figure 1B) [38]. Anesthesia was maintained with $2 \%$ isoflurane (RWD, Shenzhen, China) in nitrous oxide/oxygen (70:30). Briefly, the internal carotid artery (ICA) and pterygopalatine artery of the ICA were carefully isolated. The left external carotid artery (ECA) was ligated far away from the bifurcation. To occlude the ICA, a $0.38 \mathrm{~mm}$ nylon filament (Cinontech Co., Beijing, China) was inserted from the left common carotid artery (CCA) to the ICA through a small incision and then advanced approximately $18 \mathrm{~mm}$ into the Circle of Willis until a faint resistance was felt. Successful pMCAO (at least 70\% reduction of MCA flow compared to baseline) was confirmed by laser Doppler flowmetry (PeriFlux System 5000; Perimed, Stockholm, Sweden). Sham-operated rats underwent the same surgical procedure except for suture insertion. The rats were returned to their heated cages with free access to water and food after operation. After specific ischemic time, the available animals were further selected according to the neurological score. The rats were returned to their heated cages with free access to water and food after operation. After specific ischemic time, the available animals were further selected according to the neurological score. The neurological deficits were assessed by Zea longa test in a blinded manner as previously described [65]. 0 : no neurological deficit; 1 : failure to extend left forepaw fully; 2: circling to the left; 3 : falling to the left; 4 : failure to walk spontaneously or no consciousness; 5 : death. Rats with neurological scores of 1-4 were enrolled for further treatment. All drugs were administered by intravenous injection at according time points of ischemia. There was no sample size difference between the beginning and end of the experiments.

\subsection{TTC Staining and Behavior Assessment}

TTC (Sigma-Aldrich, St. Louis, MO, USA) staining was used for determining the infarct areas as previously described [66]. Brains were removed instantly at $3 \mathrm{~h}$ post drug administration, and six consecutive coronal slices $(2 \mathrm{~mm}$ ) were prepared and stained with $1.5 \%$ TTC in phosphate-buffered saline (PBS) for $15 \mathrm{~min}$ and then fixed in $4 \%$ formaldehyde overnight. The infarct area was analyzed using IMAGE-PRO PLUS 6.0 (Media Cybernetics, Silver Springs, MD, USA) software: infarct area $(\%)=$ total infarct area/total section area $\times 100 \%$; edema ratio $(\%)=$ left brain area/right brain area $\times 100 \%$ [67]. The neurological deficits of rats were assessed with Zea Longa test, Grid test, hanging test, and screen test in a blinded manner.

The purpose of the grid test is to evaluate the motor ability of rats. Animals placed on an elevated $(1 \mathrm{~m})$ grid surface platform with square openings of $9 \times 9 \mathrm{~cm}$ were encouraged to traverse the grid surface for one minute. The foot faults where animals inaccurately placed a limb through one of the openings in the grid were counted. The number of foot faults made per meter in one minute was calculated. The purpose of the hanging test is to check grasping ability. A stainless steel bar $(50 \mathrm{~cm}$ in length) resting on two vertical supports and elevated $60 \mathrm{~cm}$ above a flat surface was used in this test. Rats were placed on the bar midway between the supports and were observed for $120 \mathrm{~s}$. The amount of time spent hanging was recorded. $1: \leq 10 \mathrm{~s} ; 2: 11 \mathrm{~s}-30 \mathrm{~s} ; 3: 31 \mathrm{~s}-2 \mathrm{~min} ; 4: 3 \mathrm{~min}-5 \mathrm{~min} ; 5$ : $>5 \mathrm{~min}$. Screen training: The screen is $50 \times 40 \mathrm{~cm}$ mesh belt. The mesh is $1 \times 1 \mathrm{~cm}$. The left and right sides of the stencil are made of a $25-\mathrm{cm}$ high wooden board. The height of the screen is $80 \mathrm{~cm}$ from the ground. The bottom is covered with $12-\mathrm{cm}$ thick sponge. We first placed the screen horizontally, then placed the mouse on it. We then slowly raise one end, and turn the screen into a vertical position within $2 \mathrm{~s}$, keeping it that way for $5 \mathrm{~s}$. We then observe whether the rat will come down from the screen or use the front paw to grip the screen to evaluate the gripping ability and muscle strength of the front paw. 
The scoring standard is divided into four levels: 0 : The front paws hold the screen for $5 \mathrm{~s}$ for a long time, but will not fall; 1 : temporarily hold the screen, slip a distance, but did not fall; 2 : fall within $5 \mathrm{~s}$; 3: when the screen turns, the mouse immediately falls [37].

\subsection{Magnetic Resonance Imaging (MRI)}

MRI scans were performed after designated ischemic time or $3 \mathrm{~h}$ post drug administration. The animals were anaesthetized with $2.5 \%$ isoflurane and fixed in a body restrainer with tooth-bar in an MRI spectrometer (PharmaScan 70/16, Bruker, Germany). Their brains were scanned using a rat head surface coil. Diffusion weighted imaging (DWI) was used with the following parameters: echo time: $22 \mathrm{~ms}$; repetition time: $2500 \mathrm{~ms}$; slice thickness: $0.5 \mathrm{~mm}$; image size: $128 \times 128$; field of view: $25 \times 25 \mathrm{~mm}$; B value: $650 \mathrm{~s} / \mathrm{mm}^{2}$. Forty successive coronal images were acquired, from which the same position pictures in all the group were selected. Hyperintense infarct areas in DWI images were assigned with a region of interest tool and analyzed using Image-Pro Plus 6.0 software in a blinded manner: infarct area $(\%)=$ total infarct area/total section area $\times 100 \%$; edema ratio $(\%)=$ left brain area/right brain area $\times 100 \%[66]$.

\subsection{ELISA Analysis}

After anesthesia, the brain, heart and lung of rats were dissected out. Hippocampus, middle cortex and striatum of the infarcted hemisphere were freed from other parts. The tissues were homogenized with cold physiological saline $(1 \mathrm{mg}$ : $9 \mathrm{~mL}$ ). The homogenate was centrifuged (5000 rpm for $15 \mathrm{~min}$ at $4{ }^{\circ} \mathrm{C}$ ) to collect supernatants for determination of interleukin $1 \beta$ (IL-1 $\beta$ ), tumor necrosis factor $\alpha$ (TNF- $\alpha$ ) and lactate dehydrogenase (LDH) activity with ELISA kits (Nanjing Jiancheng Bioengineering Institute, Nanjing, China) according to manufacturer's instructions in a blinded manner. Quantification of ELISA results was performed at $450 \mathrm{~nm}$ in a microplate reader (Thermo Scientific, Waltham, MA, USA).

\subsection{Nissl Staining}

Nissl staining was performed with Cresyl Violet, which can selectively stain the Nissl body in survival neurons [35]. Rats were sacrificed at $3 \mathrm{~h}$ post drug administration and then immediately perfused by cold PBS (0.1 M; pH 7.4) and 4\% paraformaldehyde. The brain was removed and immersed in fixative. Then, the tissues were embedded in paraffin. Paraffin sections $(3 \mu \mathrm{m})$ were cut on glass slides, stained with $1 \%$ Cresyl Violet dissolved in $0.25 \%$ acetic acid, and examined under a light microscope (CKX41, Olympus, Tokyo, Japan) by a pathologist blinded to the study groups $(40 \times$ and $400 \times)$. Cell counts from the left and right hippocampus, cortex, and striatum on each of the six sections were averaged to provide a single value (number of neurons per $200 \mu \mathrm{m}$ length) for each animal.

\subsection{Histo-Pathological Examination}

Rats were sacrificed and then immediately perfused with cold PBS (0.1 M; pH 7.4) and 4\% paraformaldehyde. The apex of the heart, lung were fixed in $4 \%$ paraformaldehyde, routinely processed, and embedded in paraffin. Paraffin sections $(3 \mu \mathrm{m})$ were cut on glass slides, stained with hematoxylin-eosin (HE), and examined under a light microscope (CKX41, Olympus, Tokyo, Japan) by a pathologist blinded to the study groups.

\subsection{Cardiac Function Detection}

The right carotid artery was exposed via a neck dissection. A cannulation was advanced inserted into LV (left ventricle) through right carotid artery of rats, which was connected to a RM6240B multichannel physiological signal acquisition and processing system to record the following parameters of cardiac function, including left ventricular systolic pressure (LVSP), left ventricular developed 
pressure (LVDP), the maximal ventricular pressure rise ratio during systolic period $(+\mathrm{dp} / \mathrm{dtmax})$, and the maximal ventricular pressure decrease ratio during diastolic period ( $-\mathrm{dp} / \mathrm{dtmax}$ ).

\subsection{Pulmonary Function Detection}

After endotracheal intubation, a LEAD-7000 multi-channel physiological recorder was used to record the following parameters of pulmonary function, including minute ventilation (VE) and forced expiratory volume in the first second (FEV1).

\subsection{Immunohistological Staining}

Rats were sacrificed and then immediately perfused by cold PBS (0.1 M; pH 7.4) and 4\% paraformaldehyde. The brain, apex of the heart, and lung was fixed in $4 \%$ paraformaldehyde, routinely processed, and embedded in paraffin. Tissues were cut into 3- $\mu \mathrm{m}$ thick slices. After deparaffinization, endogenous peroxidase was inactivated by $3 \% \mathrm{H} 2 \mathrm{O} 2$ for $10 \mathrm{~min}$ at $37^{\circ} \mathrm{C}$. Then, the slices were permeabilized in PBS for 5 min three times at room temperature. After blocking with $5 \%$ bovine serum albumin (BSA) for $1 \mathrm{~h}$, slices were then treated with primary antibody anti-CKLF1 (Peking University Center for Human Disease Genomics, Beijing, China; 1:200 dilution) overnight at $4{ }^{\circ} \mathrm{C}$. After washing three times in PBST (0.1\% Tween-20 in PBS), the slices were incubated with biotinylated goat anti-rabbit Abs (074-1506, KPL, Gaithersburg, MD, USA; 1:200 dilution) for $2 \mathrm{~h}$ followed by peroxidase-labeled streptavidin (KPL, Gaithersburg, MD, USA; 1:500 dilution) complex for $1 \mathrm{~h}$. The expression of CKLF1 was detected with 3, 3-diaminobenzidine (DBA) as the substrate. Slices were captured using a microscope (CKX41, Olympus, Tokyo, Japan); the numbers of positive cells were scored in ten randomly selected images of the ischemic hemisphere, and the results were expressed as the number of positive cells per image using IMAGE-PRO PLUS 6.0 (Media Cybernetics, MD, USA) in a blinded manner.

\subsection{Immunofluorescence Staining}

The brain, heart and lung were processed for making paraffin section. Slices were immersed in antigen retrieval at over $95^{\circ} \mathrm{C}$ for $10 \mathrm{~min}$ in a microwave and cooled naturally. After blocking with $5 \%$ BSA, the tissues were incubated with primary antibody to CCR4 (ab216560, Abcam, Cambridge, UK; for IF, 1:200 dilution) overnight at $4{ }^{\circ} \mathrm{C}$. Following three washes in PBST $(0.1 \%$ Tween-20 in PBS), the slices were incubated with a secondary antibody Alexa 488-conjugated donkey anti-rabbit (A21206, Invitrogen, Eugene, OR, USA; 1:500 dilution), and nuclei were stained with Hoechst 33342 (H342, DOJINDO, Kumamoto, Japan; 1:1000 dilution). The number of positive cells was counted in ten randomly selected images under an upright fluorescence microscope (Leica DFC420, Wetzlar, Germany) in a blinded manner, and the results were expressed as the number of positive cells per image $(400 \times)$.

\subsection{3. $q P C R$}

Total RNA was isolated from brain, heart, and lung using TransZol Up kit (TRANSGEN BIOTECH, Beijing, China). RNA was reverse-transcribed to cDNA using TransScript One-Step gDNA Removal (TRANSGEN BIOTECH, Beijing, China) and cDNA Synthesis SuperMix kit (TRANSGEN BIOTECH, Beijing, China). First-strand cDNA was synthesized using $1 \mu \mathrm{g}$ of total RNA. The amplification of cDNA was performed with an Applied Biosystems 7900HT Fast Real-Time PCR System (Foster City, CA, USA) using TransStart Tip Green qPCR Supermix kit (TRANSGEN BIOTECH, Beijing, China). The mRNA level of individual genes was normalized to the expression of $\beta$-actin housekeeping control gene for each sample and calculated using the $\Delta \Delta C_{\mathrm{T}}$ method. The primers used for each target gene were as follows, CKLF1 (forward-5'-CGT AGA CCA TCA GCC CTT CTG-3'; reverse-5'-TCA GGA AAC CAA ACA CCC CTC-3'); CCR4 (forward-5'-CAA CGT GGT GCT TTT CCT GG-3'; reverse-5'-CAG GGT TAA GGC AGC AGT GA-3'); IL-1 $\beta$ (forward-5'-AAT GCC TCG TGC TGT CTG A- $3^{\prime}$; reverse-5'-AAT GCC TCG TGC TGT CTG A-3'); TNF- $\alpha$ (forward-5'-ACG TCG TAG CAA 
ACC ACC AA-3'; reverse-5'-GCA GCC TTG TCC CTT GAA GA-3'); actin (forward-5'-TCA GGT CAT CAC TAT CGG CAA T-3'; reverse-5'-AAA GAA AGG GTG TAA AAC GCA-3').

\subsection{Western Blotting}

Tissues were lysated and proteins were extracted for western blot as described [68]. Tissue were lysed in RIPA lysis buffer (Beyotime, Shanghai, China) for $30 \mathrm{~min}$ on ice. After centrifugation at $12,000 \mathrm{rpm}$ for $30 \mathrm{~min}$, the supernatants were collected and protein concentrations were assessed with a BCA kit (Applygen, Beijing, China). Loading buffer was added into the supernatant to denature the protein. Fifty micrograms of proteins from animals in each test group was separated on $15 \%$ gels by electrophoresis and then transferred to polyvinylidene difluoride (PVDF) membrane (Millipore, Medford, MA, USA) in blotting buffer for $1 \mathrm{~h}$ at $300 \mathrm{~mA}$. The whole PVDF membrane was then blocked with $3 \%$ BSA for $2 \mathrm{~h}$ at room temperature and then incubated with primary antibodies anti-CKLF1 (Peking University Center for Human Disease Genomics, Beijing, China; 1:500 dilution), anti-CCR4 (ab83250, Abcam, Cambridge, UK; 1:500 dilution), anti-NF-kB-p65 (sc-109, Santa Cruz Biotechnology, Santa Cruz, CA, USA; 1:500 dilution), anti-p-NF-кB-p65 (3036, Cell Signaling Technology, Beverly, MA, USA; 1:500 dilution), anti-IL-1 $\beta$ (ab9722, Abcam, Cambridge, UK; 1:500 dilution), and anti-TNF- $\alpha$ (ab6671, Abcam, Cambridge, UK; $1: 500$ dilution) at $4{ }^{\circ} \mathrm{C}$ overnight. $\beta$-actin (ab8226, Abcam, Cambridge, UK; 1:5000 dilution) was measured as loading control. Then secondary antibodies affinity purified antibody peroxidase-labeled goat anti-rabbit IgG H and L (KPL, Gaithersburg, MD, USA) and peroxidase-labeled streptavidin (074-1506, KPL, Gaithersburg, MD, USA; dilution, 1:2000) were incubated for two hours at room temperature with shaking. The expression of each protein was detected with enhanced chemiluminescence (ECL) plus detection system (Molecular Device, Lmax). The density of each band was quantified using image analysis software Gel-Pro Analyzer [69].

\subsection{Statistical Analysis}

All statistical analysis were performed using Prism (7.0; GraphPad Software, La Jolla, CA, USA). All of the data were expressed as the mean \pm standard deviation (SD). Data were analyzed using the Student's t-test or one-way ANOVA followed by the Tukey's test or were analyzed using two-way ANOVA followed by Bonferroni's multiple comparison test with Prism 7.00 software. Statistical significance was considered as $p<0.05$. The number of rats used in each in vivo condition is indicated in the corresponding figure legends.

\section{Conclusions}

In the present study, IMM-H004 was found to have protective effects against permanent focal cerebral ischemia induced brain injury and cardiopulmonary complications in aged rats. CKLF1 is an important mediator showed significant increase after ischemia in brain, heart and lung, and this chemokine is necessary for the IMM-H004 to exert protective effects. IMM-H004 inhibits the expression of CKLF1, suppressing the followed inflammatory response, and further protects the brain, heart, and lung from damage. These findings may have implications for CKLF1 as an important mediator in occurrence of cardiopulmonary complications after ischemic stroke, and also provide new ideas for drug development on ischemic stroke, especially the stroke induced cardiopulmonary complications therapy.

Supplementary Materials: Supplementary materials can be found at http:/ / www.mdpi.com/1422-0067/20/7/ $1661 / \mathrm{s} 1$.

Author Contributions: Q.A., S.C., Z.Z., S.Z., and N.C. conceptualized and designed the study; Q.A., C.C., P.Y., Y.G., X.Z., and N.C. acquired and analyzed the data; Q.A., C.C., Y.L., and N.C. drafted the text and prepared the figures. Final approval of the version to be published: All authors.

Funding: This work was supported by Hunan University of Chinese Medicine First-Class Disciple Construction Project (201803), Project of NDRC and State Administration of Traditional Chinese Medicine (ZYBZH-Y-HUN-24), Hunan Engineering Technology Center of Standardization and Function of Chinese Herbal Decoction Pieces 
(2016TP2008), Open fund of Hunan Engineering Technology Center of Standardization and Function of Chinese Herbal Decoction Pieces (BG201701), the Hunan Provincial Key Laboratory for Standardization of Important Chinese Herbal Pieces (4981-0901020), the National Natural Science Foundation of China (81730096, 81873026, 81730093), the National Mega-project for Innovative Drugs (2018ZX09711001-002-007, 2018ZX09711001-003-005, 2018ZX09711001-009-013), the CAMS Innovation Fund for Medical Sciences (CIFMS) (2016-I2M-1-004), the Beijing Key Laboratory of New Drug Mechanisms and Pharmacological Evaluation Study (BZ0150), the PUMC Graduate Education and Teaching Reform Project (10023201600801), and the opening Program of Shanxi Key Laboratory of Chinese Medicine Encephalopathy (CME-OP-2017001).

Conflicts of Interest: The authors declare no conflict of interest.

\section{Abbreviations}

\begin{tabular}{|c|c|}
\hline CKLF1 & Chemokine-like factor 1 \\
\hline CCR4 & C-C chemokine receptor type 4 \\
\hline TTC & 2,3,5-triphenyltetrazolium-chloride \\
\hline MRI & Magnetic resonance imaging \\
\hline ELISA & Enzyme-linked immunosorbent assay \\
\hline qPCR & Quantitative real-time PCR \\
\hline tPA & Tissue plasminogen activator \\
\hline FDA & Food and Drug Administration \\
\hline MI & Myocardial infarction \\
\hline PE & Pulmonary embolism \\
\hline $\mathrm{tMCAO}$ & Transient middle cerebral artery occlusion \\
\hline pMCAO & Permanent middle cerebral artery occlusion \\
\hline $\mathrm{ICA}$ & Internal carotid artery \\
\hline ECA & External carotid artery \\
\hline CCA & Common carotid artery \\
\hline PBS & Phosphate-buffered saline \\
\hline DWI & Diffusion weighted imaging \\
\hline IL-1 $\beta$ & Interleukin $1 \beta$ \\
\hline $\mathrm{TNF}-\alpha$ & Tumor necrosis factor $\alpha$ \\
\hline LDH & Lactate dehydrogenase \\
\hline BCA & Bicinchoninic acid \\
\hline $\mathrm{HE}$ & Hematoxylin-eosin \\
\hline LVSP & Left ventricular systolic pressure \\
\hline LVDP & Left ventricular developed pressure \\
\hline$+\mathrm{dp} / \mathrm{dtmax}$ & The maximal ventricular pressure rise ratio during systolic period \\
\hline$-\mathrm{dp} / \mathrm{dtmax}$ & The maximal ventricular pressure decrease ratio during diastolic period \\
\hline VE & Minute ventilation \\
\hline FEV1 & Forced expiratory volume in the first second \\
\hline BSA & Bovine serum albumin \\
\hline DBA & 3,3-diaminobenzidin \\
\hline PVDF & Polyvinylidene difluoride \\
\hline ECL & Enhanced chemiluminescence \\
\hline SD & Standard deviation. \\
\hline
\end{tabular}

\section{References}

1. Feigin, V.L.; Forouzanfar, M.H.; Krishnamurthi, R.; Mensah, G.A.; Connor, M.; Bennett, D.A.; Moran, A.E.; Sacco, R.L.; Anderson, L.; Truelsen, T.; et al. Global and regional burden of stroke during 1990-2010: Findings from the Global Burden of Disease Study 2010. Lancet 2014, 383, 245-254. [CrossRef]

2. Benjamin, E.J.; Virani, S.S.; Callaway, C.W.; Chamberlain, A.M.; Chang, A.R.; Cheng, S.; Chiuve, S.E.; Cushman, M.; Delling, F.N.; Deo, R.; et al. Heart Disease and Stroke Statistics-2018 Update: A Report From the American Heart Association. Circulation 2018, 137, e67-e492. [CrossRef] [PubMed] 
3. Rojas, J.I.; Zurru, M.C.; Romano, M.; Patrucco, L.; Cristiano, E. Acute ischemic stroke and transient ischemic attack in the very old-Risk factor profile and stroke subtype between patients older than 80 years and patients aged less than 80 years. Eur. J. Neurol. 2007, 14, 895-899. [CrossRef] [PubMed]

4. Leonardi-Bee, J.; Bath, P.M.; Phillips, S.J.; Sandercock, P.A. Blood pressure and clinical outcomes in the International Stroke Trial. Stroke 2002, 33, 1315-1320. [CrossRef] [PubMed]

5. Williams, G.R.; Jiang, J.G.; Matchar, D.B.; Samsa, G.P. Incidence and occurrence of total (first-ever and recurrent) stroke. Stroke 1999, 30, 2523-2528. [CrossRef]

6. Appelros, P.; Stegmayr, B.; Terent, A. Sex differences in stroke epidemiology: A systematic review. Stroke 2009, 40, 1082-1090. [CrossRef] [PubMed]

7. Morris, D.C. Thrombolysis 3 to 4.5 Hours after Acute Ischemic Stroke. N. Engl. J. Med. 2008, 359, $2839-2841$.

8. Sun, Y.Y.; Morozov, Y.M.; Yang, D.; Li, Y.; Dunn, R.S.; Rakic, P.; Chan, P.H.; Abe, K.; Lindquist, D.M.; Kuan, C.Y. Synergy of combined tPA-edaravone therapy in experimental thrombotic stroke. PLoS ONE 2014, 9, e98807. [CrossRef]

9. Won, S.; Lee, J.K.; Stein, D.G. Recombinant tissue plasminogen activator promotes, and progesterone attenuates, microglia/macrophage M1 polarization and recruitment of microglia after MCAO stroke in rats. Brain Behav. Immun. 2015, 49, 267-279. [CrossRef] [PubMed]

10. Johnston, K.C.; Li, J.Y.; Lyden, P.D.; Hanson, S.K.; Feasby, T.E.; Adams, R.J.; Faught, R.E., Jr.; Haley, E.C., Jr. Medical and neurological complications of ischemic stroke: Experience from the RANTTAS trial. RANTTAS Investigators. Stroke 1998, 29, 447-453. [CrossRef]

11. Hong, K.S.; Kang, D.W.; Koo, J.S.; Yu, K.H.; Han, M.K.; Cho, Y.J.; Park, J.M.; Bae, H.J.; Lee, B.C. Impact of neurological and medical complications on 3-month outcomes in acute ischaemic stroke. Eur. J. Neurol. 2008, 15, 1324-1331. [CrossRef] [PubMed]

12. Viitanen, M.; Winblad, B.; Asplund, K. Autopsy-verified causes of death after stroke. Acta Med. Scand. 1987, 222, 401-408. [CrossRef] [PubMed]

13. Balami, J.S.; Chen, R.L.; Grunwald, I.Q.; Buchan, A.M. Neurological complications of acute ischaemic stroke. Lancet Neurol. 2011, 10, 357-371. [CrossRef]

14. Kumar, S.; Selim, M.H.; Caplan, L.R. Medical complications after stroke. Lancet Neurol. 2010, 9, 105-118. [CrossRef]

15. Vernino, S.; Brown, R.D., Jr.; Sejvar, J.J.; Sicks, J.D.; Petty, G.W.; O'Fallon, W.M. Cause-specific mortality after first cerebral infarction: A population-based study. Stroke 2003, 34, 1828-1832. [CrossRef] [PubMed]

16. Bronnum-Hansen, H.; Davidsen, M.; Thorvaldsen, P. Long-term survival and causes of death after stroke. Stroke 2001, 32, 2131-2136. [CrossRef] [PubMed]

17. Hardie, K.; Hankey, G.J.; Jamrozik, K.; Broadhurst, R.J.; Anderson, C. Ten-year survival after first-ever stroke in the perth community stroke study. Stroke 2003, 34, 1842-1846. [CrossRef] [PubMed]

18. Koennecke, H.C.; Belz, W.; Berfelde, D.; Endres, M.; Fitzek, S.; Hamilton, F.; Kreitsch, P.; Mackert, B.M.; Nabavi, D.G.; Nolte, C.H.; et al. Factors influencing in-hospital mortality and morbidity in patients treated on a stroke unit. Neurology 2011, 77, 965-972. [CrossRef] [PubMed]

19. Iadecola, C.; Anrather, J. The immunology of stroke: From mechanisms to translation. Nat. Med. 2011, 17, 796-808. [CrossRef]

20. Chamorro, A.; Hallenbeck, J. The harms and benefits of inflammatory and immune responses in vascular disease. Stroke 2006, 37, 291-293. [CrossRef]

21. Offner, H.; Vandenbark, A.A.; Hurn, P.D. Effect of experimental stroke on peripheral immunity: CNS ischemia induces profound immunosuppression. Neuroscience 2009, 158, 1098-1111. [CrossRef] [PubMed]

22. Liu, Q.; Jin, W.N.; Liu, Y.; Shi, K.; Sun, H.; Zhang, F.; Zhang, C.; Gonzales, R.J.; Sheth, K.N.; La Cava, A.; et al. Brain Ischemia Suppresses Immunity in the Periphery and Brain via Different Neurogenic Innervations. Immunity 2017, 46, 474-487. [CrossRef] [PubMed]

23. Katzan, I.L.; Cebul, R.D.; Husak, S.H.; Dawson, N.V.; Baker, D.W. The effect of pneumonia on mortality among patients hospitalized for acute stroke. Neurology 2003, 60, 620-625. [CrossRef] [PubMed]

24. Yang, G.Y.; Chen, X.; Sun, Y.C.; Ma, C.L.; Qian, G. Chemokine-like factor 1 (CLFK1) is over-expressed in patients with atopic dermatitis. Int. J. Biol. Sci. 2013, 9, 759-765. [CrossRef] [PubMed] 
25. Zheng, Y.; Guo, C.; Zhang, Y.; Qi, H.; Sun, Q.; Xu, E.; Zhang, Y.; Ma, D.; Wang, Y. Alleviation of murine allergic rhinitis by C19, a C-terminal peptide of chemokine-like factor 1 (CKLF1). Int. Immunopharmacol. 2011, 11, 2188-2193. [CrossRef] [PubMed]

26. Tan, Y.X.; Yang, T.; Chen, Q.L.; Ye, F.; Jiang, M.; Li, S.Y.; Ding, J.; Song, Q.S.; Li, X.T.; Tang, Y.; et al. Outcome and significance of pulmonary pathological changes induced by a single intramuscular injection of chemokine-like factor 1 in mice. Zhonghua Yi Xue Za Zhi. 2009, 89, 2408-2411.

27. Li, G.; Wang, D.; Sun, M.; Li, G.; Hu, J.; Zhang, Y.; Yuan, Y.; Ji, H.; Chen, N.; Liu, G. Discovery and optimization of novel 3-piperazinylcoumarin antagonist of chemokine-like factor 1 with oral antiasthma activity in mice. J. Med. Chem. 2010, 53, 1741-1754. [CrossRef] [PubMed]

28. Kong, L.L.; Hu, J.F.; Zhang, W.; Yuan, Y.H.; Ma, K.L.; Han, N.; Chen, N.H. Expression of chemokine-like factor 1 after focal cerebral ischemia in the rat. Neurosci. Lett. 2011, 505, 14-18. [CrossRef] [PubMed]

29. Kong, L.L.; Hu, J.F.; Zhang, W.; Yuan, Y.H.; Han, N.; Chen, N.H. C19, a C-terminal peptide of chemokine-like factor 1, protects the brain against focal brain ischemia in rats. Neurosci. Lett. 2012, 508, 13-16. [CrossRef] [PubMed]

30. Kong, L.L.; Wang, Z.Y.; Hu, J.F.; Yuan, Y.H.; Han, N.; Li, H.; Chen, N.H. Inhibition of chemokine-like factor 1 protects against focal cerebral ischemia through the promotion of energy metabolism and anti-apoptotic effect. Neurochem. Int. 2014, 76, 91-98. [CrossRef] [PubMed]

31. Santos Samary, C.; Pelosi, P.; Leme Silva, P.; Rieken Macedo Rocco, P. Immunomodulation after ischemic stroke: Potential mechanisms and implications for therapy. Crit. Care 2016, 20, 391. [CrossRef] [PubMed]

32. Ishikawa, H.; Tajiri, N.; Vasconcellos, J.; Kaneko, Y.; Mimura, O.; Dezawa, M.; Borlongan, C.V. Ischemic stroke brain sends indirect cell death signals to the heart. Stroke 2013, 44, 3175-3182. [CrossRef] [PubMed]

33. Wang, Y.; Zhang, Y.; Han, W.; Li, D.; Tian, L.; Yin, C.; Ma, D. Two C-terminal peptides of human CKLF1 interact with the chemokine receptor CCR4. Int. J. Biochem. Cell Biol. 2008, 40, 909-919. [CrossRef] [PubMed]

34. Sun, M.; Hu, J.; Song, X.; Wu, D.; Kong, L.; Sun, Y.; Wang, D.; Wang, Y.; Chen, N.; Liu, G. Coumarin derivatives protect against ischemic brain injury in rats. Eur. J. Med. Chem. 2013, 67, 39-53. [CrossRef] [PubMed]

35. Zuo, W.; Zhang, W.; Han, N.; Chen, N.H. Compound IMM-H004, a novel coumarin derivative, protects against CA1 cell loss and spatial learning impairments resulting from transient global ischemia. CNS Neurosci. Ther. 2015, 21, 280-288. [CrossRef] [PubMed]

36. Niu, F.; Song, X.Y.; Hu, J.F.; Zuo, W.; Kong, L.L.; Wang, X.F.; Han, N.; Chen, N.H. IMM-H004, A New Coumarin Derivative, Improved Focal Cerebral Ischemia via Blood-Brain Barrier Protection in Rats. J. Stroke Cerebrovasc. Dis. 2017, 26, 2065-2073. [CrossRef] [PubMed]

37. Yang, P.-F.; Song, X.-Y.; Zeng, T.; Ai, Q.-D.; Liu, D.-D.; Zuo, W.; Zhang, S.; Xia, C.-Y.; He, X.; Chen, N.-H. IMM-H004, a coumarin derivative, attenuated brain ischemia/reperfusion injuries and subsequent inflammation in spontaneously hypertensive rats through inhibition of VCAM-1. RSC Adv. 2017, 7, 27480-27495. [CrossRef]

38. Zuo, W.; Chen, J.; Zhang, S.; Tang, J.; Liu, H.; Zhang, D.; Chen, N. IMM-H004 prevents toxicity induced by delayed treatment of $\mathrm{tPA}$ in a rat model of focal cerebral ischemia involving PKA-and PI3K-dependent Akt activation. Eur. J. Neurosci. 2014, 39, 2107-2118. [CrossRef] [PubMed]

39. Zhang, G.; Zhang, R.; Wu, H.; Wang, H.; Zhang, W.; Zhang, L.; Sun, H.; Li, Y.; Bu, N.; Du, Y.; et al. Recanalization of occluded large arteries with broadened therapeutic window for acute cerebral infarction. Clin. Neurol. Neurosurg. 2013, 115, 1009-1015. [CrossRef] [PubMed]

40. Jung, S.; Gralla, J.; Fischer, U.; Mono, M.L.; Weck, A.; Ludi, R.; Heldner, M.R.; Findling, O.; El-Koussy, M.; Brekenfeld, C.; et al. Safety of endovascular treatment beyond the 6-h time window in 205 patients. Eur. J. Neurol. 2013, 20, 865-871. [CrossRef] [PubMed]

41. Yang, J.; Cui, X.; Li, J.; Zhang, C.; Zhang, J.; Liu, M. Edaravone for acute stroke: Meta-analyses of data from randomized controlled trials. Dev. Neurorehabil. 2015, 18, 330-335. [CrossRef] [PubMed]

42. Wu, H.Y.; Tang, Y.; Gao, L.Y.; Sun, W.X.; Hua, Y.; Yang, S.B.; Zhang, Z.P.; Liao, G.Y.; Zhou, Q.G.; Luo, C.X.; et al. The synergetic effect of edaravone and borneol in the rat model of ischemic stroke. Eur. J. Pharmacol. 2014, 740, 522-531. [CrossRef] [PubMed]

43. Oguro, H.; Mitaki, S.; Takayoshi, H.; Abe, S.; Onoda, K.; Yamaguchi, S. Retrospective Analysis of Argatroban in 353 Patients with Acute Noncardioembolic Stroke. J. Stroke Cerebrovasc. Dis. 2018, 27, 2175-2181. [CrossRef] [PubMed] 
44. Watanabe, K.; Tanaka, M.; Yuki, S.; Hirai, M.; Yamamoto, Y. How is edaravone effective against acute ischemic stroke and amyotrophic lateral sclerosis? J. Clin. Biochem. Nutr. 2018, 62, 20-38. [CrossRef]

45. Jin, R.; Liu, L.; Zhang, S.; Nanda, A.; Li, G. Role of inflammation and its mediators in acute ischemic stroke. J. Cardiovasc. Transl. Res. 2013, 6, 834-851. [CrossRef] [PubMed]

46. Kong, L.L.; Wang, Z.Y.; Han, N.; Zhuang, X.M.; Wang, Z.Z.; Li, H.; Chen, N.H. Neutralization of chemokine-like factor 1 , a novel C-C chemokine, protects against focal cerebral ischemia by inhibiting neutrophil infiltration via MAPK pathways in rats. J. Neuroinflamm. 2014, 11, 112. [CrossRef] [PubMed]

47. Kong, L.L.; Wang, Z.Y.; Hu, J.F.; Yuan, Y.H.; Li, H.; Chen, N.H. Inhibition of chemokine-like factor 1 improves blood-brain barrier dysfunction in rats following focal cerebral ischemia. Neurosci. Lett. 2016, 627, 192-198. [CrossRef]

48. Shim, R.; Wong, C.H. Ischemia, Immunosuppression and Infection-Tackling the Predicaments of Post-Stroke Complications. Int. J. Mol. Sci. 2016, 17, 64. [CrossRef] [PubMed]

49. Liu, D.D.; Chu, S.F.; Chen, C.; Yang, P.F.; Chen, N.H.; He, X. Research progress in stroke-induced immunodepression syndrome (SIDS) and stroke-associated pneumonia (SAP). Neurochem. Int. 2018, 114, 42-54. [CrossRef] [PubMed]

50. Aslanyan, S.; Weir, C.J.; Diener, H.C.; Kaste, M.; Lees, K.R. Pneumonia and urinary tract infection after acute ischaemic stroke: A tertiary analysis of the GAIN International trial. Eur. J. Neurol. 2004, 11, 49-53. [CrossRef] [PubMed]

51. Hilker, R.; Poetter, C.; Findeisen, N.; Sobesky, J.; Jacobs, A.; Neveling, M.; Heiss, W.D. Nosocomial pneumonia after acute stroke: Implications for neurological intensive care medicine. Stroke 2003, 34, 975-981. [CrossRef] [PubMed]

52. Liesz, A.; Hagmann, S.; Zschoche, C.; Adamek, J.; Zhou, W.; Sun, L.; Hug, A.; Zorn, M.; Dalpke, A.; Nawroth, P.; et al. The spectrum of systemic immune alterations after murine focal ischemia: Immunodepression versus immunomodulation. Stroke 2009, 40, 2849-2858. [CrossRef]

53. Prass, K.; Meisel, C.; Hoflich, C.; Braun, J.; Halle, E.; Wolf, T.; Ruscher, K.; Victorov, I.V.; Priller, J.; Dirnagl, U.; et al. Stroke-induced immunodeficiency promotes spontaneous bacterial infections and is mediated by sympathetic activation reversal by post-stroke $\mathrm{T}$ helper cell type 1-like immunostimulation. J. Exp. Med. 2003, 198, 725-736. [CrossRef]

54. Williams, J.L.; Holman, D.W.; Klein, R.S. Chemokines in the balance: Maintenance of homeostasis and protection at CNS barriers. Front. Cell. Neurosci. 2014, 8, 154. [CrossRef] [PubMed]

55. Mukaida, N.; Matsumoto, T.; Yokoi, K.; Harada, A.; Matsushima, K. Inhibition of neutrophil-mediated acute inflammation injury by an antibody against interleukin-8 (IL-8). Inflamm. Res. 1998, 47 (Suppl. 3), S151-S157. [CrossRef] [PubMed]

56. Lawrence, T. The nuclear factor NF-kappaB pathway in inflammation. Cold Spring Harb. Perspect. Biol. 2009, 1, a001651. [CrossRef] [PubMed]

57. Shih, R.H.; Wang, C.Y.; Yang, C.M. NF-kappaB Signaling Pathways in Neurological Inflammation: A Mini Review. Front. Mol. Neurosci. 2015, 8, 77. [CrossRef]

58. Clemens, J.A.; Stephenson, D.T.; Dixon, E.P.; Smalstig, E.B.; Mincy, R.E.; Rash, K.S.; Little, S.P. Global cerebral ischemia activates nuclear factor-kappa B prior to evidence of DNA fragmentation. Brain Res. Mol. Brain Res. 1997, 48, 187-196. [CrossRef]

59. Venna, V.R.; Weston, G.; Benashski, S.E.; Tarabishy, S.; Liu, F.; Li, J.; Conti, L.H.; McCullough, L.D. NF-kappaB contributes to the detrimental effects of social isolation after experimental stroke. Acta Neuropathol. 2012, 124, 425-438. [CrossRef]

60. Chauhan, A.; Moser, H.; McCullough, L.D. Sex differences in ischaemic stroke: Potential cellular mechanisms. Clin. Sci. (Lond.) 2017, 131, 533-552. [CrossRef]

61. Zuo, W.; Zhang, W.; Chen, N.H. Sexual dimorphism in cerebral ischemia injury. Eur. J. Pharmacol. 2013, 711, 73-79. [CrossRef] [PubMed]

62. Gibson, C.L. Cerebral ischemic stroke: Is gender important? J. Cereb. Blood Flow Metab. 2013, 33, $1355-1361$. [CrossRef] [PubMed]

63. Bekhbat, M.; Neigh, G.N. Sex differences in the neuro-immune consequences of stress: Focus on depression and anxiety. Brain Behav. Immun. 2018, 67, 1-12. [CrossRef] [PubMed]

64. Klein, S.L.; Flanagan, K.L. Sex differences in immune responses. Nat. Rev. Immunol. 2016, 16, 626-638. [CrossRef] [PubMed] 
65. Longa, E.Z.; Weinstein, P.R.; Carlson, S.; Cummins, R. Reversible middle cerebral artery occlusion without craniectomy in rats. Stroke 1989, 20, 84-91. [CrossRef] [PubMed]

66. Zhang, S.; Song, X.-Y.; Xia, C.-Y.; Ai, Q.-D.; Chen, J.; Chu, S.-F.; He, W.-B.; Chen, N.-H. Effects of cerebral glucose levels in infarct areas on stroke injury mediated by blood glucose changes. RSC Adv. 2016, 6, 93815-93825. [CrossRef]

67. Zhang, S.; Qi, Y.; Xu, Y.; Han, X.; Peng, J.; Liu, K.; Sun, C.K. Protective effect of flavonoid-rich extract from Rosa laevigata Michx on cerebral ischemia-reperfusion injury through suppression of apoptosis and inflammation. Neurochem. Int. 2013, 63, 522-532. [CrossRef] [PubMed]

68. Zuo, W.; Yang, P.F.; Chen, J.; Zhang, Z.; Chen, N.H. Drp-1, a potential therapeutic target for brain ischaemic stroke. Br. J. Pharmacol. 2016, 173, 1665-1677. [CrossRef]

69. Liu, S.; Ai, Q.; Feng, K.; Li, Y.; Liu, X. The cardioprotective effect of dihydromyricetin prevents ischemia-reperfusion-induced apoptosis in vivo and in vitro via the PI3K/Akt and HIF-1alpha signaling pathways. Apoptosis 2016, 21, 1366-1385. [CrossRef] [PubMed]

(C) 2019 by the authors. Licensee MDPI, Basel, Switzerland. This article is an open access article distributed under the terms and conditions of the Creative Commons Attribution (CC BY) license (http:// creativecommons.org/licenses/by/4.0/). 